Atmos. Chem. Phys., 13, 3945-3977, 2013

www.atmos-chem-phys.net/13/3945/2013/

doi:10.5194/acp-13-3945-2013

(C) Author(s) 2013. CC Attribution 3.0 License.

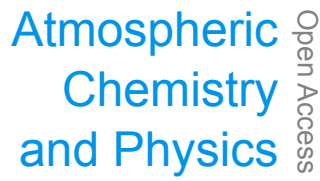

\title{
Recent variability of the solar spectral irradiance and its impact on climate modelling
}

\author{
I. Ermolli ${ }^{1}$, K. Matthes ${ }^{2}$, T. Dudok de Wit ${ }^{3}$, N. A. Krivova ${ }^{4}$, K. Tourpali ${ }^{5}$, M. Weber ${ }^{6}$, Y. C. Unruh ${ }^{7}$, L. Gray ${ }^{8}$, \\ U. Langematz ${ }^{9}$, P. Pilewskie ${ }^{10}$, E. Rozanov ${ }^{11,12}$, W. Schmutz ${ }^{11}$, A. Shapiro ${ }^{11}$, S. K. Solanki ${ }^{4,13}$, and T. N. Woods ${ }^{10}$ \\ ${ }^{1}$ INAF, Osservatorio Astronomico di Roma, Monte Porzio Catone, Italy \\ ${ }^{2}$ GEOMAR I Helmholtz-Zentrum für Ozeanforschung Kiel, Kiel, Germany \\ ${ }^{3}$ LPC2E, CNRS and University of Orléans, Orléans, France \\ ${ }^{4}$ Max-Planck-Institut für Sonnensystemforschung, 37191 Katlenburg-Lindau, Germany \\ ${ }^{5}$ Laboratory of Atmospheric Physics, Aristotle University of Thessaloniki, Greece \\ ${ }^{6}$ Institut für Umweltphysik, Universität Bremen FB1, Bremen, Germany \\ ${ }^{7}$ Astrophysics Group, Blackett Laboratory, Imperial College London, SW7 2AZ, UK \\ ${ }^{8}$ Centre for Atmospheric Sciences, Dept. of Atmospheric, Oceanic and Planetary Physics, University of Oxford, UK \\ ${ }^{9}$ Institut für Meteorologie, Freie Universität Berlin, Berlin, Germany \\ ${ }^{10}$ University of Colorado, Laboratory for Atmospheric and Space Physics, Boulder, CO, USA \\ ${ }^{11}$ Physikalisch-Meteorologisches Observatorium, World Radiation Center, Davos Dorf, Switzerland \\ ${ }^{12}$ IAC ETH, Zurich, Switzerland \\ ${ }^{13}$ School of Space Research, Kyung Hee University, Yongin, Gyeonggi 46-701, Republic of Korea \\ Correspondence to: I. Ermolli (ilaria.ermolli@oa-roma.inaf.it)
}

Received: 29 July 2012 - Published in Atmos. Chem. Phys. Discuss.: 19 September 2012

Revised: 6 March 2013 - Accepted: 13 March 2013 - Published: 17 April 2013

\begin{abstract}
The lack of long and reliable time series of solar spectral irradiance (SSI) measurements makes an accurate quantification of solar contributions to recent climate change difficult. Whereas earlier SSI observations and models provided a qualitatively consistent picture of the SSI variability, recent measurements by the SORCE (SOlar Radiation and Climate Experiment) satellite suggest a significantly stronger variability in the ultraviolet (UV) spectral range and changes in the visible and near-infrared (NIR) bands in anti-phase with the solar cycle. A number of recent chemistry-climate model (CCM) simulations have shown that this might have significant implications on the Earth's atmosphere. Motivated by these results, we summarize here our current knowledge of SSI variability and its impact on Earth's climate.

We present a detailed overview of existing SSI measurements and provide thorough comparison of models available to date. SSI changes influence the Earth's atmosphere, both directly, through changes in shortwave (SW) heating and therefore, temperature and ozone distributions in the stratosphere, and indirectly, through dynamical feedbacks. We in-
\end{abstract}

vestigate these direct and indirect effects using several stateof-the art CCM simulations forced with measured and modelled SSI changes. A unique asset of this study is the use of a common comprehensive approach for an issue that is usually addressed separately by different communities.

We show that the SORCE measurements are difficult to reconcile with earlier observations and with SSI models. Of the five SSI models discussed here, specifically NRLSSI (Naval Research Laboratory Solar Spectral Irradiance), SATIRE-S (Spectral And Total Irradiance REconstructions for the Satellite era), COSI (COde for Solar Irradiance), SRPM (Solar Radiation Physical Modelling), and OAR (Osservatorio Astronomico di Roma), only one shows a behaviour of the UV and visible irradiance qualitatively resembling that of the recent SORCE measurements. However, the integral of the SSI computed with this model over the entire spectral range does not reproduce the measured cyclical changes of the total solar irradiance, which is an essential requisite for realistic evaluations of solar effects on the Earth's climate in CCMs. 
We show that within the range provided by the recent SSI observations and semi-empirical models discussed here, the NRLSSI model and SORCE observations represent the lower and upper limits in the magnitude of the SSI solar cycle variation.

The results of the CCM simulations, forced with the SSI solar cycle variations estimated from the NRLSSI model and from SORCE measurements, show that the direct solar response in the stratosphere is larger for the SORCE than for the NRLSSI data. Correspondingly, larger UV forcing also leads to a larger surface response.

Finally, we discuss the reliability of the available data and we propose additional coordinated work, first to build composite SSI data sets out of scattered observations and to refine current SSI models, and second, to run coordinated CCM experiments.

\section{Introduction}

The question of whether - and to what extent - the Earth's climate is influenced by solar variability remains central to the understanding of anthropogenic climate change. According to the 4th assessment report of the Intergovernmental Panel on Climate Change, solar variability represents about $8 \%$ of recent total net radiative anthropogenic forcing (Solomon et al., 2007). However, there is a large uncertainty in this figure because several aspects of solar forcing and the different mechanisms by which solar variability influences the Earth's environment are still poorly understood (see e.g. Pap et al., 2004; Calisesi et al., 2007; Haigh, 2007; Gray et al., 2010; Lockwood, 2012, and references therein). For these reasons, the quantification of solar contribution to climate change remains incomplete. This is further highlighted by some of the most recent investigations of solar spectral irradiance (SSI) variations and estimates of their influence on the Earth's atmosphere based on chemistry-climate model (CCM) simulations.

Regular space-based measurements of the solar irradiance started in 1978. The total solar irradiance (TSI), i.e. the spectrally integrated radiative power density of the Sun incident at the top of Earth's atmosphere, has been monitored almost continuously and was found to vary on different time scales (Willson et al., 1981; Fröhlich and Lean, 2004; Fröhlich, 2009). Most noticeable is the $\approx 0.1 \%$ modulation of TSI in phase with the $11 \mathrm{yr}$ solar cycle. Changes 2-3 times larger than this are observed on timescales shorter than a few days. Measurements of SSI, however, are not continuous over the satellite era and until recently have concentrated on the ultraviolet (UV) radiation, because of the larger relative variability of SSI below $400 \mathrm{~nm}$ (Fig. 1) and the impact of these wavelengths on the terrestrial atmosphere through radiative heating and ozone photochemistry.

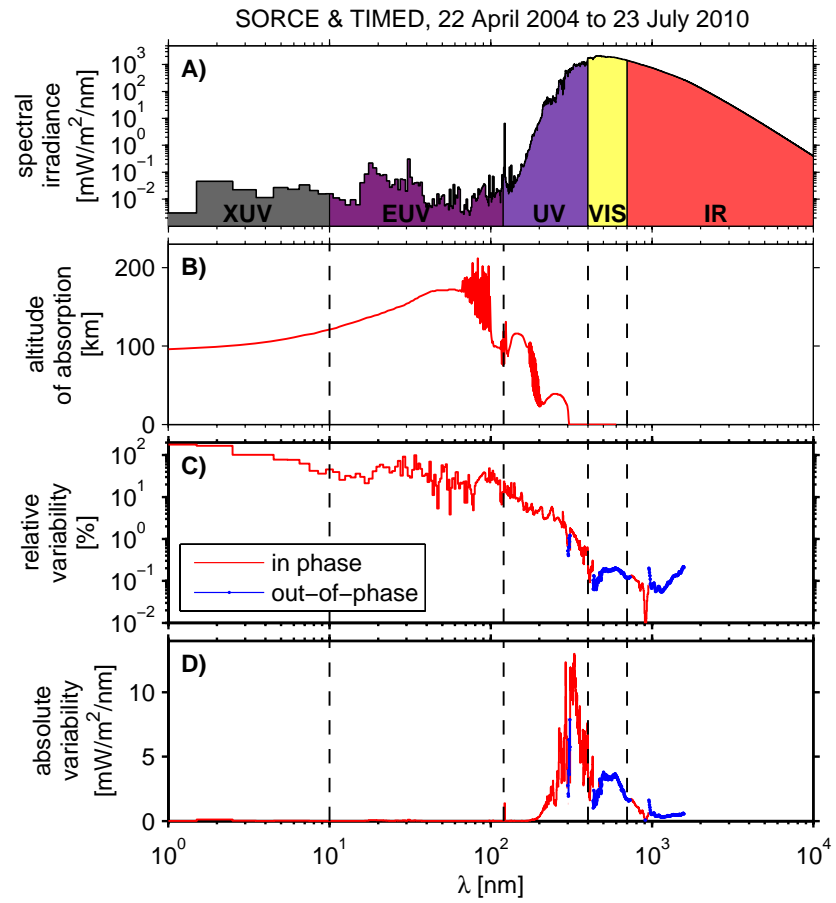

Fig. 1. The solar spectral irradiance as inferred from SORCE and TIMED observations only, from 22 April 2004 till 23 July 2010. (A) shows the average solar spectral irradiance for that period. A black-body model has been used to extend the SSI for wavelengths beyond $1580 \mathrm{~nm}$. (B) displays the characteristic altitude of absorption in the Earth's atmosphere for each wavelength, defined as the altitude at which the optical depth equals one. (C) shows the relative variability (peak to peak/average) for solar cycle variations inferred from measurements obtained between 22 April 2004 and 23 July 2010. Spectral regions, where the variability is in phase with the solar cycle (represented by, e.g. the sunspot number or the TSI) are marked in red, while blue denotes ranges where the variability measured by SORCE is out-of-phase with the solar cycle. These phases, as well as the magnitude of the variability in the UV, are not all reproduced by models and other observations (see Sect. 3 as well as Figs. 2, 7 and 8), and thus should be considered with care. (D) shows the absolute variability, which peaks strongly in the near-UV.

SSI variations differ from those observed in the TSI. The variability of visible and NIR (near-infrared) bands barely exceeds $0.5 \%$ over a solar cycle; in the near UV and shorter wavelengths variability increases with decreasing wavelength, reaching several percent at $200-250 \mathrm{~nm}$, and several tens of percent, and even more, below about $200 \mathrm{~nm}$ (e.g. Floyd et al., 2003, and references therein). These bands are almost completely absorbed in the Earth's middle and upper atmosphere (Fig. 1) and are the primary agent affecting heating, photochemistry, and therefore, the dynamics of the Earth's atmosphere. Variations of the solar UV radiation between 120 and $350 \mathrm{~nm}$ lead to changes in stratospheric ozone and heating that amplify the effect of the UV radiation in 


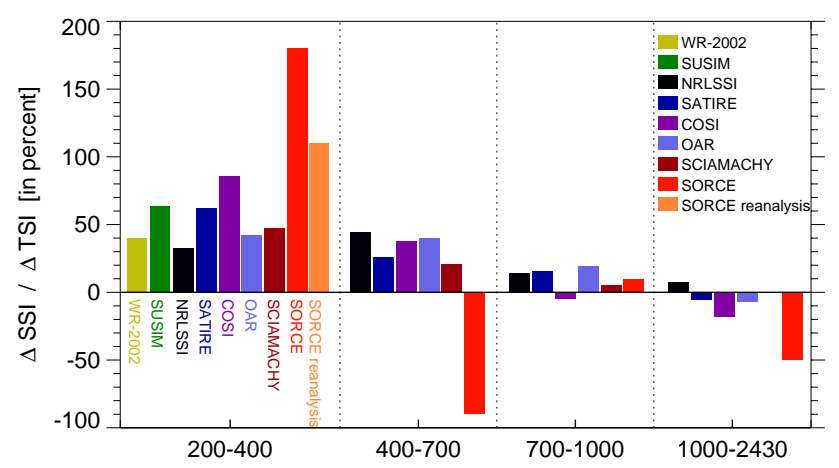

Fig. 2. Relative contribution of the UV (200-400 nm), visible (400$700 \mathrm{~nm})$, near-IR (700-1000 $\mathrm{nm})$ and IR (1000-2430 nm) ranges to the TSI change over the solar cycle as derived from measurements and models described in Sects. 2 and 3. For SORCE/SIM, only the period between 2004 and 2008 can be considered. For other data and models, the plotted relative differences are between solar maximum an minimum. Within each wavelength bin, from left to right: WR-2002 (light green, only in UV; Woods and Rottman, 2002), UARS/SUSIM (green, only in UV; Morrill et al., 2011a), NRLSSI (black), SATIRE-S (blue), COSI (purple), OAR (light blue), SCIAMACHY (brown), SORCE (red), and SORCE re-analysis (orange, only in UV; Woods, 2012). The exact wavelength ranges used for SUSIM and SCIAMACHY in the UV are $150-400 \mathrm{~nm}$ and 240 $400 \mathrm{~nm}$, respectively. The possible related corrections are, however, expected to lie within 2-3\%. Note that for the SCIAMACHY-based model, the original values listed by Pagaran et al. (2009) are shown. As discussed by Krivova and Solanki (2013), these values should most likely be corrected by a factor of roughly 1.2 .

the Earth's atmosphere, possibly also through indirect mechanisms (e.g. the "top-down" mechanism, Gray et al., 2010). Hence, although the UV radiation shortward of $400 \mathrm{~nm}$ represents less than $8 \%$ of the TSI, its variability may have a significant impact on climate. In contrast, the visible and IR bands, which have the largest contribution to the TSI, small variations over the solar cycle, and no absorption in the atmosphere but in some well-defined IR bands, directly heat the Earth's surface and the lower atmosphere. The large amount of solar flux at the visible and IR bands implies that small flux differences may induce important terrestrial consequences. The impact of the variability of these bands on the Earth's climate is expected to be small, although it may involve amplification mechanisms (e.g. the "bottom-up" mechanism, van Loon et al., 2007; Meehl et al., 2009).

Early satellite measurements of the solar UV variability have shown a qualitatively consistent behaviour (DeLand and Cebula, 2012), which is fairly well reproduced by SSI models (e.g. Lean et al., 1997; Krivova et al., 2006; Unruh et al., 2012; Lean and DeLand, 2012). This situation changed with the launch of the Spectral Irradiance Monitor instrument (SIM, Harder et al., 2005a) onboard the Solar Radiation and Climate Experiment satellite (SORCE, Rottman, 2005 ) in 2003, which was shortly after the most recent max- imum of solar activity. The SORCE/SIM data showed a 4-6 times greater decrease of the UV radiation between 200 and $400 \mathrm{~nm}$ over the period 2004-2008 (Harder et al., 2009), part of the declining phase of solar cycle 23, compared to earlier measurements and models (Ball et al., 2011; Pagaran et al., 2011a; Unruh et al., 2012; DeLand and Cebula, 2012; Lean and DeLand, 2012). This larger decrease measured in the UV (Fig. 2), which exceeds the TSI decrease over the same period by almost a factor of two, is compensated by an increase in the visible and NIR bands. Variability out-of-phase with solar activity is indeed predicted by some SSI models in the NIR, but with a significantly lower magnitude than found by SORCE/SIM. Details are provided in the following. The inverse variability observed by SIM in a wide integrated band in the visible was, however, unexpected. It can be interpreted as a result of effects induced by the evolution of surface magnetism in the solar atmosphere (e.g. Harder et al., 2009). However, other observations and analyses of existing longterm SSI data show results in contrast with those derived from SORCE/SIM (Wehrli et al., 2012).

When used as solar input to CCM simulations, SORCE/SIM observations lead to significantly larger shortwave (SW) heating rates in the upper stratosphere compared to results obtained by using the commonly utilised NRLSSI (Naval Research Laboratory Solar Spectral Irradiance) model data (Lean et al., 1997; Lean, 2000), and a decrease of stratospheric ozone above an altitude of $45 \mathrm{~km}$ during solar maximum (Haigh et al., 2010). These changes in radiative heating and ozone photochemistry in the stratosphere also impact the responses of the "top-down" solar UV mechanism in the Earth's atmosphere and at the surface (Kodera and Kuroda, 2002), which may depart from current understanding (Cahalan et al., 2010; Ineson et al., 2011; Merkel et al., 2011; Oberländer et al., 2012; Swartz et al., 2012). Validation of the results of these model simulations with ozone, zonal wind, or temperature measurements is difficult, because the data are sparse and do not cover enough solar cycles. Although some ozone observations seem to agree with model calculations (e.g. Haigh et al., 2010; Merkel et al., 2011), it should be noted that the SIM measurements employed for the analysis covered less than one solar cycle and required extrapolation over a full cycle, and therefore, added uncertainty (Garcia, 2010). Also the transition altitude from in-phase (lower and middle stratosphere) to out-ofphase (upper stratosphere) ozone signals with the solar cycle is not consistent among the different models and requires further investigations.

Unfortunately, observations of the full solar spectrum will likely have a multi-year gap before the next generation SSI instrument is launched. Based on data presently available, a thorough understanding of the impact of SSI on climate requires verification and validation of existing SSI measurements for internal consistency, calculations of middleatmosphere climate models with different reliable scenarios of SSI variations, and comparison of measurements and 
model results with climate records, i.e. a study involving the coordinated work of various research communities, which is part of the COST (Cooperation in Science and Technology) Action ES1005 ${ }^{1}$ to which most of the authors of this paper belong.

This paper summarises and compares, for the first time, a large number of SSI observations and models, and discusses the impact of these data on Earth's climate. A review in this area was recently published by Gray et al. (2010), but their focus was more on different possible forcing mechanisms. Although the Sun affects the climate system in numerous ways, we here focus on radiative forcing only, with particular attention given to the role of the SSI rather than that of the TSI, which is still the sole solar input in many climate models. Since space-based observations of the SSI and of the terrestrial atmosphere conditions are sparse or absent before 1980, we restrict our analysis to the data of the last three decades, i.e. roughly the last three solar cycles. Furthermore, we concentrate on the effects of the UV variability because of its potentially large impact on the terrestrial atmosphere.

The paper is organised as follows: in Sect. 2, measurements of SSI variations are described, and their accuracy on timescales from days to $11 \mathrm{yr}$ solar cycle is discussed. In Sect. 3 we delineate mechanisms responsible for the SSI variations, outline methods of irradiance reconstructions and briefly describe and compare several of the most broadly used models of SSI variations. Section 4 discusses the impact of the current lower and upper boundaries of SSI solar cycle estimated variations on the atmospheric response in several current state-of-the art CCMs. A summary and concluding remarks are provided in Sect. 5.

\section{Solar irradiance measurements}

We first discuss the available solar irradiance measurements, focussing on the most recent data. These data are input to and, at the same time, the main source of constraints for both the SSI models and CCM simulations presented below. We describe the evolution of measurements carried out from space since 1978 and discuss major instrumental and measurement problems that limit the creation of single composite time series from existing records (Sect. 2.1). Long and accurate SSI time series are critical to obtain reliable estimates of the solar variability impact on the terrestrial atmosphere. We then present instruments and techniques used to derive four SSI data sets that are employed for CCM simulations (Sect. 2.2). Finally, we discuss recent re-assessment of the TSI absolute value (Sect. 2.3), since it enters some climate and SSI models, and outline the perspectives of future SSI observations (Sect. 2.4).

\footnotetext{
${ }^{1}$ TOSCA - towards a more complete assessment of the impact of solar variability on the Earth's climate, http://www.tosca-cost.eu.
}

\subsection{Observation of solar irradiance}

For many centuries, the Sun has been considered as an example of stability and, not surprisingly, the TSI, i.e. the quantity of radiative power density $\left(\mathrm{W} \mathrm{m}^{-2}\right)$ at normal incidence on top of the atmosphere, at a Sun-Earth distance of one astronomical unit, has been called solar constant. This term is still often used. However, regular space-based observations that started in 1978 (Willson et al., 1981) have revealed that the TSI varies over timescales of minutes to decades, and probably even longer (e.g. Fröhlich, 2006, 2009).

The most noticeable variation of the TSI is a $0.1 \%$ modulation in phase with the solar activity or sunspot cycle. Although quantifying such small variations is a major technological challenge, it is strongly motivated by the desire to understand solar variability, and even more so by the importance of the TSI for terrestrial energy budget studies (Trenberth et al., 2009).

The TSI is the spectral integral of SSI over all wavelengths but its weak variability masks the fact that relative SSI variations show a strong wavelength dependence (Fig. 1). In particular, the visible and NIR bands are the least variable of the solar spectrum with a relative solar cycle amplitude of the same order as for the TSI $(0.1 \%)$, whereas values of 1 to $100 \%$ are observed in the UV variations, and in excess of $100 \%$ in the soft X-ray range (below $10 \mathrm{~nm}$ ). Each individual spectral band has a markedly different impact on the terrestrial atmosphere, which depends on the atmospheric processes affected by the given band, the amount of the spectral flux, and its variation.

Figure 3 shows an overview of all SSI space experiments as a function of the period of observations and wavelength coverage (above $100 \mathrm{~nm}$ ). Regular space-based monitoring of the solar spectrum over a broad range, covering, in addition to the UV, the visible and the IR up to $2.4 \mu \mathrm{m}$, started with the launch of ENVISAT/SCIAMACHY (European ENVIronmental SATellite/SCanning Imaging Absorption spectroMeter for Atmospheric CHartographY) in 2002 and SORCE/SIM in 2003. Measurements in the UV below $400 \mathrm{~nm}$ began several decades earlier. Most of these earlier measurements, however, are difficult to use for the quantification of the solar variability, for reasons that will be detailed below.

The longest records of SSI measurements were provided by SOLSTICE (SOLar STellar Irradiance Comparison Experiment) and SUSIM (Solar Ultraviolet Spectral Irradiance Monitor) aboard the UARS (Upper Atmosphere Research Satellite) spacecraft (Rottman et al., 2004). These instruments observed solar UV radiation between 120 and $400 \mathrm{~nm}$ from 1991 to 2001 and 2005, respectively. These measurements pointed to the importance of the irradiance variations in the UV (Woods et al., 2000; Rottman et al., 2001; Floyd et al., 2002, 2003), although the solar cycle variability of solar radiation above approximately $250 \mathrm{~nm}$ remained relatively uncertain due to insufficient long-term stability of the 


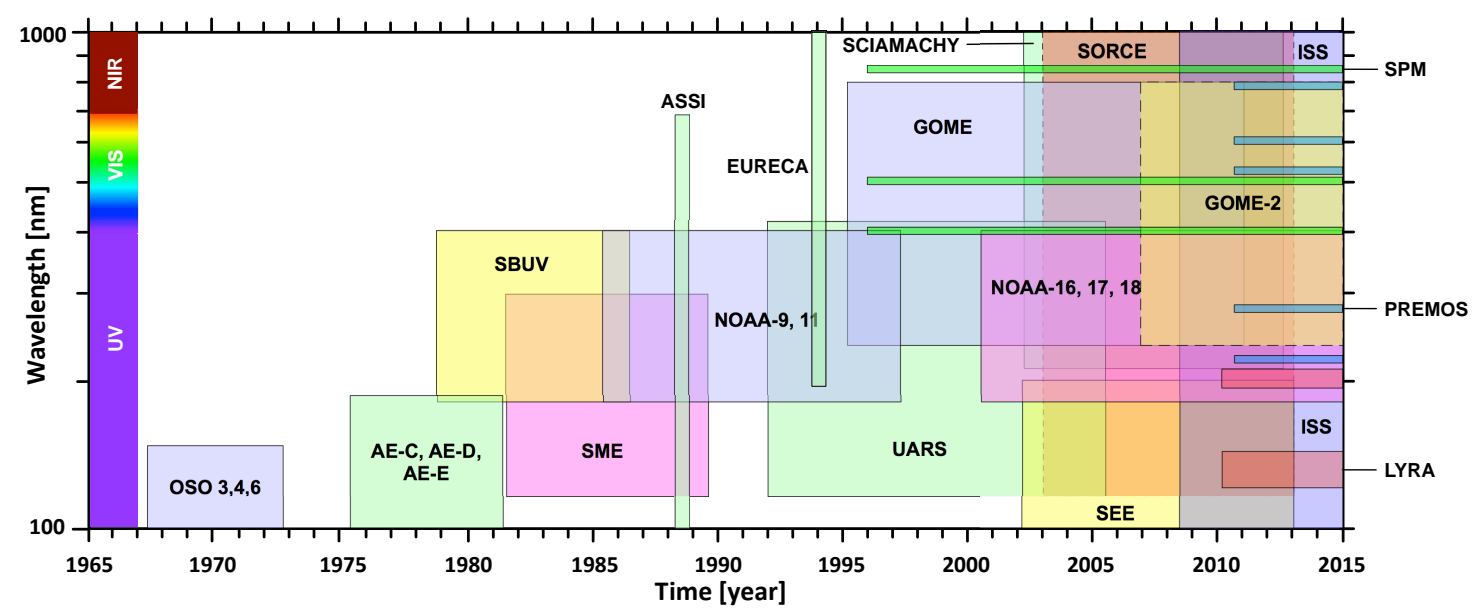

Fig. 3. Overview of the main satellite missions that have made SSI observations at wavelengths higher than $100 \mathrm{~nm}$. Short missions such as sounding rockets are not indicated.

instruments (Woods et al., 1996). In addition to SOLSTICE and SUSIM, there is the long time series of $200-400 \mathrm{~nm}$ solar UV observations by several NOAA SBUV (Solar Backscatter Ultraviolet) instruments, which had underflight calibrations aboard the Space Shuttle (Cebula et al., 1998). Table 1 of DeLand and Cebula (2012) summarises the measurement uncertainties for these instruments.

Regular observations of the visible and NIR bands covering more than one year started with SOHO/VIRGO-SPM (SOlar and Heliospheric Observatory/Variability of IRradiance and Gravity Oscillations Sun PhotoMeter) (Fröhlich et al., 1997) in 1996 at three selected bands and continued with the ERS/GOME (European Remote Sensing/Global Ozone Monitoring Experiment) (Weber et al., 1998; Burrows et al., 1999), ENVISAT/SCIAMACHY (Bovensmann et al., 1999), SORCE/SIM (Harder et al., 2005a,b), and ISS/SOLSPEC (International Space Station/SOLar SPECtrum) (Thuillier et al., 2009) instruments. These SSI missions are described in more detail below.

Merging all UV observations into a single homogeneous composite record is a major challenge (DeLand et al., 2004) that is hampered by several problems. First, the lifetime of most instruments does not exceed a decade. This makes longterm observations covering periods that exceed single instrument lifetimes, of prime interest for climate models, very difficult. A second obstacle is the differing technologies and modes of operation of various space-based instruments. The cross-calibration of individual records is further hampered by the fact that overlapping observations disagree, and the existing data records are spectrally and temporally intermittent. Although missing observations can be filled in by using data regression based on time series of solar proxies such as the Mg II index, which are well correlated with UV variations (DeLand and Cebula, 1993; Viereck et al., 2001; Lean, 1997), none of the existing solar proxies can properly repro- duce solar irradiance in a spectral band on all timescales (Dudok de Wit et al., 2009).

The most critical issue for all SSI instruments is the optical degradation caused by the energetic radiation in the space environment. Two options have been employed to account for instrumental degradations. The first one is to provide redundancy in the instrument design, by using, e.g. a dual spectrometer set-up with detectors that experience different accumulated exposure time or by planning redundancies in spectral channels and calibration lamps from which degradation corrections can be derived. This approach was used, for instance, for UARS/SUSIM and SORCE/SIM (Brueckner et al., 1993; Harder et al., 2005a). The second option is to use reference data, e.g. solar irradiance measurements for long-term calibrations, as reported for the NOAA SBUV instruments, periodic recalibrations using sounding rockets, or stable external calibration targets like selected stars, as done for UARS/SOLSTICE and SORCE/SOLSTICE (McClintock et al., 2005). Sensitivity changes and degradation are strongly wavelength-dependent, which makes creating a properly cross-calibrated SSI record very difficult. One attempt for a composite SSI time series in the UV is provided by DeLand and Cebula (2008).

Cross-calibration of different SSI records is also limited by the lack of realistic confidence intervals for the existing data. This aspect has been thoroughly investigated for the TSI and sound estimates have been obtained for timescales from days to years. No meaningful estimates, however, exist for timescales exceeding one decade, which are needed for climate studies. The situation is much worse for SSI measurements, whose relative uncertainties often are two to three orders of magnitude larger.

In the following subsections a brief summary of newly available SSI observations obtained during the last decade is given. Also a brief description of recent developments 
regarding the absolute values of the TSI, and its variability is provided.

\subsection{SSI time series}

\subsubsection{SCIAMACHY and GOME}

SCIAMACHY (SCanning Imaging Absorption spectroMeter for Atmospheric CHartographY) and GOME (Global Ozone Monitoring Experiment) onboard the ENVISAT and ERS-2 satellites, respectively, are atmospheric sounders that measure terrestrial atmospheric trace gases (Burrows et al., 1999; Bovensmann et al., 1999; Bovensmann et al., 2011). The primary purpose of direct solar measurements by SCIAMACHY and the two GOMEs (a second GOME is flying on MetOp-A (Meteorological Operation) since 2006 and a third GOME on MetOp-B since 2012) is to Sun-normalise the backscattered light from the terrestrial atmosphere, which is then inverted to determine atmospheric trace gas amounts. This normalisation does not require absolute radiometric calibration and cancels out degradation effects. The SCIAMACHY and GOME instruments have been radiometrically calibrated before launch.

In order to provide estimates for solar cycle variability from SCIAMACHY measurements $(230 \mathrm{~nm}-2.4 \mu \mathrm{m})$ without the need for a detailed degradation correction, a proxy model (hereafter referred to as SCIAMACHY proxy model) was developed by fitting solar proxy time series to observed SCIAMACHY measurements over several 27 day solar rotation periods (Pagaran et al., 2009). The proxies used in this model are the photometric sunspot index (Balmaceda et al., 2009) and the Mg II index (Weber et al., 2013) for sunspot darkening and facular brightening. Assuming that the fitting parameters linearly scale from solar rotations to an $11 \mathrm{yr}$ solar cycle, one can then use the solar proxies to extrapolate beyond the lifetime of the single instrument (Pagaran et al., 2011b). Note, however, that this assumption might not be accurate, and probably results in an underestimate of the magnitude of the solar cycle variation. The short-term variability of the SCIAMACHY proxy model over several solar rotations agrees well with direct solar observations from SCIAMACHY, SORCE/SIM, and UARS/SUSIM (Pagaran et al., 2009, 2011a). Over longer periods during the descending phases of solar cycles 21 to 23 , larger differences between model and direct observations become apparent. In particular, SORCE/SIM data show UV changes that are about four times larger than the SCIAMACHY proxy model (Pagaran et al., 2011b; Fig. 2).

Very recently an optical degradation model has been developed that uses the various light paths from different combinations of mirrors within SCIAMACHY. The main causes for the optical degradation are believed to be contaminants on the mirror surfaces. This new degradation model with improved calibration corrections should allow us, in the near future, to derive long-term time series of SCIAMACHY measurements without the use of the SCIAMACHY proxy model.

\subsubsection{SORCE}

The SOlar Radiation and Climate Experiment (SORCE; Rottman, 2005), launched in January 2003, has made continuous daily measurements of SSI from 0.1 to $2400 \mathrm{~nm}$ (with missing portions of the extreme UV between $35 \mathrm{~nm}$ and $115 \mathrm{~nm}$ ), accounting for about $97 \%$ of the TSI. These measurements are unique compared to SSI existing data. TSI is also measured on SORCE by the Total Irradiance Monitor (TIM; Kopp et al., 2005). The two instruments onboard SORCE pertinent to this study are the Solar Stellar Irradiance Comparison Experiment (SOLSTICE; McClintock et al., 2005) and the Spectral Irradiance Monitor (SIM; Harder et al., 2005a,b).

SORCE/SOLSTICE is a grating spectrometer that measures SSI in the UV from $115 \mathrm{~nm}$ to $320 \mathrm{~nm}$ with a resolution of $0.1 \mathrm{~nm}$ and with an absolute calibration uncertainty of approximately $5 \%$ and measurement precision to better than $0.5 \%$ on all timescales (Snow et al., 2005). SORCE/SOLSTICE is a second generation of UARS/SOLSTICE (Rottman et al., 1993), which acquired UV measurements from 1991 to 2001. SOLSTICE uses nighttime observations of stars to track and correct for changes in responsivity. SORCE/SOLSTICE uses two channels to cover the spectral regions $115-180 \mathrm{~nm}$ and 170 $320 \mathrm{~nm}$. The long-term stability in the latest data version is about $1 \%$ per year (M. Snow, personal communication, 2012).

SORCE/SIM (Harder et al., 2005a,b) was developed to replace the longest wavelength channel $(280-420 \mathrm{~nm})$ in the original UARS/SOLSTICE and to extend wavelength coverage well out into the NIR. SORCE/SIM employs a single optical element, a Féry prism, for dispersion and to focus light on four detectors in the focal plane. Two photodiode detectors cover the range from $200 \mathrm{~nm}$ to $950 \mathrm{~nm}$, another covers the range from $895 \mathrm{~nm}$ to $1620 \mathrm{~nm}$, and an electrical substitution radiometer (ESR) operates over the spectral range from $258 \mathrm{~nm}$ to $2423 \mathrm{~nm}$. This ESR is also used to calibrate the other three. Because SORCE/SIM is a prism spectrometer its resolution varies from less than $1 \mathrm{~nm}$ in UV to approximately $40 \mathrm{~nm}$ in the NIR. SORCE/SIM began reporting daily SSI results in April 2004. It achieves an absolute calibration uncertainty of approximately $2 \%$ and measurement precision of $0.1 \%$ or better at most wavelengths. Merkel et al. (2011) stated that the SIM long-term uncertainty in the $200-300 \mathrm{~nm}$ region is $\approx 0.5-0.1 \%$, from $310-400 \mathrm{~nm}$ it is $\approx 0.2-0.05 \%$, and in the $400-1600 \mathrm{~nm}$ range it is better than $0.05 \%$. However, they also reported the lack of independent observations for direct validation of these estimates. Moreover, the values above are relative to annual changes. Therefore, they can be assumed valid for short-term variations, but 
on longer timescales, the instrument stability could be considerably lower.

It is worth mentioning that the SORCE public Level 3 data include the SOLSTICE data up to $308 \mathrm{~nm}$ and SIM data above $308 \mathrm{~nm}$. However, the SIM spectra do extend down to about $200 \mathrm{~nm}$. Although the SIM data between 200$308 \mathrm{~nm}$ are not publicly available yet, they have been made exploitable for several studies through personal communications. The SORCE data used in our study to estimate the atmospheric response to SSI solar cycle variations are specified in Table 3. They also include data received by personal communications with instrument team members (J. Harder, personal communication, 2012).

Harder et al. (2009) presented multi-year SORCE/SIM trends indicating that UV variability during the declining phase of solar cycle 23 (between 2004 and 2008) was larger than that observed in previous cycles, and was compensated by trends in other bands in the visible and NIR that increased with decreasing solar activity. SORCE/SOLSTICE has also shown enhanced UV variability for the same time period. Solar UV variability measured by both SORCE/SIM and SORCE/SOLSTICE exceed the variability observed by UARS/SOLSTICE and UARS/SUSIM over solar cycle 22 and ascending phase of cycle 23 by a factor of 3-10 depending on wavelength (DeLand and Cebula, 2012; Figs. 2, 4, and 8).

These discrepancies with prior cycle observations and with SSI models have inspired new analyses and collaborations aimed at a better understanding of the potential sources of instrument degradation that might have affected SORCE instruments and previous instruments as well. The studies have been concentrated on SSI instrument observations, capabilities, and estimated spectral irradiance uncertainties, methods of correcting for degradation, and refinement of estimated uncertainties. It has been understood that all detectors and optics suffer some degradation in space, largely due to exposure to solar light, and also due to hydrocarbon contamination that dominates below $400 \mathrm{~nm}$. Accordingly, new models of degradation based on total dose, rather than just exposure time, are being developed for the SORCE and other instruments. Revised data sets, which e.g. will include SIM data down to $240 \mathrm{~nm}$, are expected out in 2013. Besides, degradation trends have also been analysed by considering the expected invariance of SSI over the solar cycle minimum. The latter method has been developed by Woods (2012) and applied to data during last solar cycle minimum (2008-2009) to estimate possible degradation trends for SORCE/SIM and SORCE/SOLSTICE. It consists of identifying near-identical solar activity levels on both sides of the minimum to derive corrections for instrument degradation. This analysis showed good agreement of the variability from moderate solar activity level to minimum level from various measurements and models, from $120 \mathrm{~nm}$ to $300 \mathrm{~nm}$ for solar cycles 21 through 24. However, as the method has about $30 \%$ uncertainty in variability due to the assumptions about selecting times of similar irradiance levels, the results may not be as accurate as those derived from analyses based on instrument degradation alone.

The analysis by Woods (2012) reduces the variability of the integrated UV irradiance from $200 \mathrm{~nm}$ to $400 \mathrm{~nm}$, relative to the measured TSI change, to $110 \%$ (Fig. 2) from the $190 \%$ change reported by Harder et al. (2009). Nevertheless, to be compatible with the observed TSI changes even this lower amplitude of the UV variation over the solar cycle still requires compensation from out-of-phase trends at other wavelengths, in particular above $\approx 400 \mathrm{~nm}$. Other analyses of solar cycle variability suggest that the UV variability in the $200 \mathrm{~nm}$ to $400 \mathrm{~nm}$ range is about $60 \%$ of the measured TSI change (Krivova et al., 2006; Pagaran et al., 2009; Morrill et al., 2011b).

\subsubsection{SOLSPEC}

The SOLar SPECtrum instrument (SOLSPEC; Thuillier et al., 2009) is composed of three double monochromators $(170-390 \mathrm{~nm}, 380-850 \mathrm{~nm}, 800-3000 \mathrm{~nm})$ and a set of lamps allowing corrections for aging related to the harsh space environment. The SOLSPEC spectrometer flew several times on the Space Shuttle and its twin instrument, SOSP (SOlar SPectrum), was placed on the EURECA (EUropean REtrieval CArrier) platform for 10 months (April 1993 to January 1994). These missions have provided data to build the ATLAS (ATmospheric Laboratory for Applications and Science) spectra, specifically ATLAS1 (March 1992) and ATLAS-3 (November 1994) (Thuillier et al., 2004), which are composites using UARS/SUSIM and UAR/SOLSTICE data from Lyman- $\alpha$ at $121 \mathrm{~nm}$ to $200 \mathrm{~nm}$, and ATLAS/SSBUV, ATLAS/SUSIM, and ATLAS/SOLSPEC from 200 to $400 \mathrm{~nm}$, ATLAS/SOLSPEC from 400 to $850 \mathrm{~nm}$, and EURECA/SOSP from 800 to $2400 \mathrm{~nm}$. The ATLAS spectra were calibrated to absolute radiometric scale using the blackbody of the Heidelberg Observatory, and tungsten and deuterium lamps calibrated by NIST (The National Institute of Standards and Technology).

SOLSPEC has been upgraded for operations onboard the International Space Station (ISS) by implementing several changes in the electronics, optics, and mechanisms, and by adding redundant components in order to generate data with proper degradation correction. ISS/SOLSPEC has been calibrated to an absolute scale at the Physikalisch-Technische Bundesanstalt (PTB) using the BB3200pg blackbody radiator (Sperfeld et al., 1998). Over the whole spectral range, SOLSPEC accuracy is within 2 to $3 \%$. The SOLSPEC spectrometer has been in operation since February 2008 onboard the ISS along with the SOL-ACES (SOLar Auto-Calibrating EUV/UV Spectrophotometers) instrument measuring below $150 \mathrm{~nm}$. When the ISS orientation allows, ISS/SOLSPEC records the solar spectral irradiance. Presently, data have been obtained during the solar minimum preceding solar cycle 24 and at some specific periods during its rising phase for 
direct comparisons with SORCE/SIM observations (Thuillier et al., 2012).

\subsubsection{Statistical analysis of SSI time series}

As mentioned above, the assessment of long-term variations in SSI observations can be done only by stitching together different records that, individually, often do not last for more than a few years and are almost always offset by different calibration scales. The first systematic effort toward building such a single composite data set for the UV was done by DeLand and Cebula (2008), who created a data set with daily spectra covering the wavelength range $120-400 \mathrm{~nm}$ for the time period November 1978 to August 2005. However, the instruments frequently differed in their radiometric calibration and in their long-term stability. One is therefore often left with making subjective adjustments that can dramatically alter the interpretation of long-term variations (Lockwood, 2011) and, for example, mimic a solar cycle variation that does not exist. Normalisation to a single reference spectrum, as done by DeLand and Cebula (2008), can only partially compensate such differences in absolute calibration. A more objective approach consists of incorporating these instrumental discrepancies in the reconstruction, and then explicitly using them as a contribution to the overall uncertainty. A Bayesian statistical framework is ideally suited for this analysis.

To illustrate this approach, we concentrate on the solar cycle (i.e. decadal) modulation, which has the advantage of being one of the conspicuous signatures of solar variability in climate records while offering sensitive means for diagnosing the quality of the solar observations.

Our analysis consists first in extending in time all SSI observations, and subsequently in analysing their $11 \mathrm{yr}$ modulation. First, for each instrument and for each wavelength, we extrapolate the SSI observations backward and forward in time while preserving their statistical properties with respect to all other observations (cross-correlation, etc.). This is made possible thanks to the empirical evidence for all neighbouring spectral bands that evolve remarkably coherently in time, on timescales of hours and beyond (Lean et al., 1982; Amblard et al., 2008). This coherency, which is rooted in the strong magnetic coupling between solar atmospheric layers, allows us to describe all salient features of the variability in the SSI with just a few degrees of freedom. There are typically three of them in the UV. The extrapolation is based on the expectation-maximisation technique (Dudok de Wit, 2011), which is routinely used to fill in missing data in climate records. As a result, we obtain for each wavelength as many records as there are instruments observing it. The overall dispersion of the various observations is then naturally reflected by the dispersion of the reconstructions, and so no offset or trend adjustments are required. We would like to highlight that no proxies are used to constrain the reconstruction.
The next step consists in comparing the $11 \mathrm{yr}$ modulation amplitude and phase for each reconstruction and identifying possible changes since 1978. To date, all spectral bands and all solar proxies show in-phase variations with each other (up to a constant pass shift), regardless of the solar cycle. This is a strong, albeit not sufficient, indication that different spectral bands are likely to remain in phase from cycle to cycle. Likewise, their modulation amplitudes are unlikely to change. Any departure from this picture should thus receive special consideration. We estimate the modulation amplitudes and phases by running an $11 \mathrm{yr}$ running mean through the data. Here, the phase reference is chosen to be the Mg II core-to-wing ratio because it is widely used as proxy for the solar UV (Viereck et al., 2001). This choice, however, has no impact on the results that follow.

Figure 4 illustrates the extrapolation for the $220-240 \mathrm{~nm}$ band, which is important for ozone production (e.g. Rozanov et al., 2002). This example shows that all observations vary in phase with the solar cycle but differ considerably in their modulation amplitude. The observations from SORCE exhibit a larger modulation amplitude, as already mentioned above. Note that the use of the MgII index as a reference is just to fix the phase reference for comparing the results obtained for different cycles, without affecting the way the SSI is extrapolated back- and forward in time.

Because this approach provides as many UV composites as there are observations, we can test whether the observations from SORCE/SIM and SORCE/SOLSTICE are compatible with those from other instruments, most of which were obtained during preceding solar cycles. The comparison is summarised in Fig. 5, which compares the modulation amplitude and phase of SORCE versus the distribution obtained by the other instruments. We conclude that all instruments agree remarkably well below $200 \mathrm{~nm}$; at longer UV wavelengths, the modulation amplitude inferred from SORCE/SOLSTICE is systematically larger by a factor of two to six at all wavelengths. The simultaneous sharp drop in its phase raises doubts about the consistency between SOLSTICE and the other measurements, and also between SIM and the other data. Assuming that there is no reason for the SSI to be unusual during the last solar cycle only, we conclude that these observations are likely to be affected by instrumental drifts, in agreement with the conclusions from Lean and DeLand (2012). This may also be the case for SORCE/SIM between 308 and $340 \mathrm{~nm}$, although the departure from other observations is much less significant here.

\subsection{TSI time series}

Some climate models still include solar variability only as derived from TSI, thereby fixing the relative spectral contribution of the solar radiation hitting the Earth. In addition, TSI time series also provide constraints for the empirical and semi-empirical models of SSI variations that are discussed in the following. Therefore, it seems appropriate to 


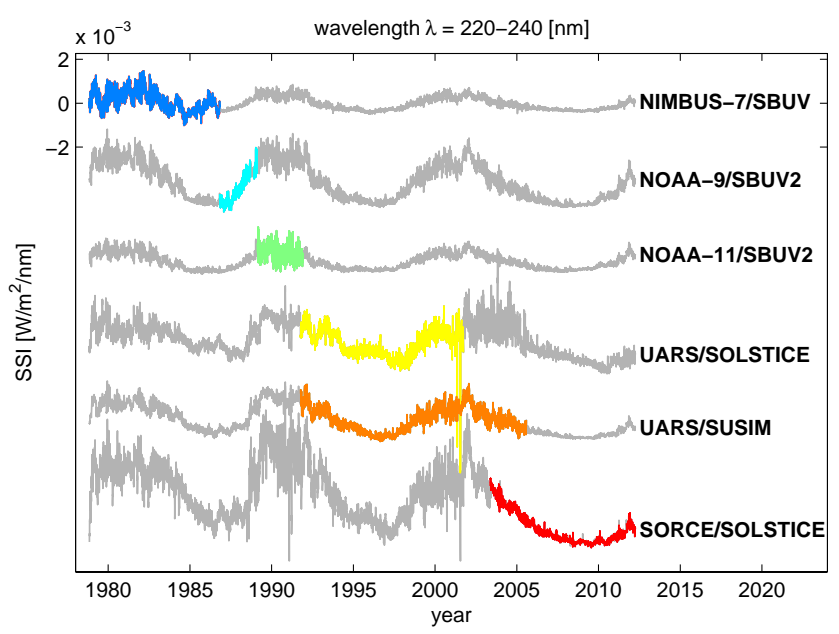

Fig. 4. Daily averages of the spectral irradiance in the $220-240 \mathrm{~nm}$ band, as measured by 6 different (and only partly overlapping) instruments. Each record has been shifted vertically for easier visualisation. Extrapolations are shown in grey, observations in colour. One- $\sigma$ confidence intervals for the former are not shown here but are typically $0.4 \times 10^{-3}\left(\mathrm{Wm}^{-2} \mathrm{~nm}^{-1}\right)$.

discuss here the current knowledge on TSI measurements. In fact, for many years, the canonical value of the average TSI was $1365.4 \pm 1.3 \mathrm{~W} \mathrm{~m}^{-2}$. Now, the most accurate, and generally accepted, value is $1361 \pm 0.5 \mathrm{~W} \mathrm{~m}^{-2}$ (Kopp and Lean, 2011; Schmutz et al., 2012). This lower value will be used in the data assimilation and meteorological re-analysis project like ERA-CLIM (ECMWF Reanalysis for climate) at ECMWF (European Centre for Medium-Range Weather Forecasts; D. Dee, ECMWF, personal communication 2012, and see http://www.era-clim.eu/).

TSI variability was already predicted in the 1920 s from ground-based observations (Abbot et al., 1923). Accurate measurement of TSI and detection of its variability requires observations from space. The first report of the variable solar irradiance with correct amplitudes was made by Hickey et al. (1980). Later observations differed markedly in their absolute values but all basically agreed in the relative amplitude of the TSI variations. In Table 1 we list the main space experiments that have measured TSI, together with their observed variabilities, which for some experiments were subsequently revised as further detailed below. The given numbers are biased by the duration of the experiments and their phase relative to the solar cycle, but the overall result is that TSI variations are observed to be on the order of about $0.5 \%$ standard deviation from the mean value.

Lee et al. (1995) estimated the absolute accuracy of the Nimbus7/HF instrument to be $0.5 \%$ and that of ERBS/ERBE (Earth Radiation Budget Satellite/Earth Radiation Budget Experiment) 0.2\%. Willson (1979) expected his SMM/ACRIM-I (Solar Maximum Mission/Active Cavity Radiometer Irradiance Monitor-I) experiment to remain

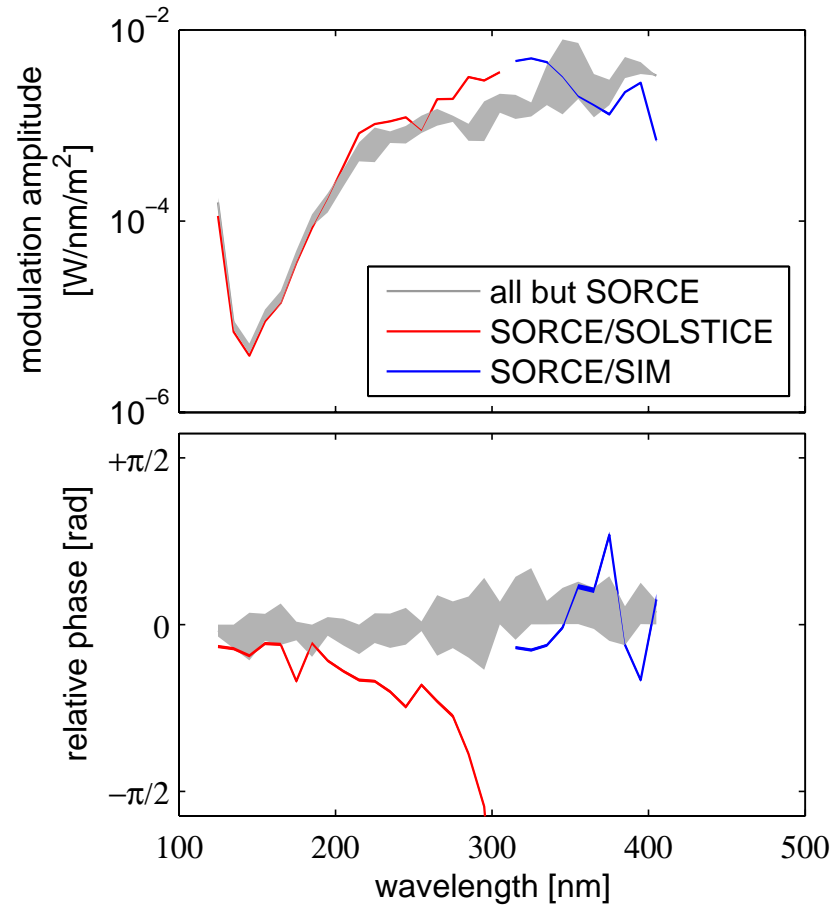

Fig. 5. Modulation amplitude (top) and phase (bottom) from SORCE/SIM and SORCE/SOLSTICE, compared to that from other instruments. The SSI has been averaged over $10 \mathrm{~nm}$ bins. The vertical width of the shaded area reflects the dispersion of the instruments ( $\pm \sigma$ confidence interval).

within $0.1 \%$ for at least a year. Fröhlich and Lean (1998) state that the absolute measurements of the early radiometers are uncertain to about $0.4 \%$, which corresponds to $5.5 \mathrm{~W} \mathrm{~m}^{-2}$. The main reason for this relatively large spread was due to the uncertainty in the aperture area. When the technology for determining aperture area improved, the agreements among the measurements improved. However, the SORCE/TIM experiment proved to be a new outlier. Lawrence et al. (2003) claim an uncertainty of $0.5 \mathrm{Wm}^{-2}$, i.e. accurate to $350 \mathrm{ppm}$. Because SORCE/TIM is 4.5 and $5 \mathrm{~W} \mathrm{~m}^{-2}$ below SOHO/VIRGO and ACRIM/ACRIM-III, respectively, the uncertainties given by the instrument teams do not overlap (Kopp and Lean, 2011).

The PREMOS (PREcision MOnitoring Sensor) experiment on the French satellite PICARD, which was launched in July 2010, has contributed to the understanding of the instrument offsets, by confirming the lower TSI value initially reported by the SORCE/TIM (Kopp et al., 2005). The radiometers of the PICARD/PREMOS experiment have been calibrated in two different and independent ways. The first is a calibration in power response as reported by Schmutz et al. (2009). In addition, the TSI radiometer of PICARD/PREMOS is, so far, the first space instrument that has been calibrated in irradiance in vacuum. This was done at the Total solar irradiance Radiometer Facility 
Table 1. Summary of main TSI measurements from space.

\begin{tabular}{llllll}
\hline Spacecraft & Instrument & Start & End & $\begin{array}{l}\text { Mean and st.dev. } \\
\left(\mathrm{W} \mathrm{m}^{-2}\right)\end{array}$ & Reference \\
\hline Nimbus & HF & Nov 1978 & Dec 1993 & $1372.1 \pm 0.8$ & Hickey et al. (1980) \\
SMM & ACRIM-I & Feb 1980 & Jul 1989 & $1367.5 \pm 0.7$ & Willson (1979) \\
ERBS & ERBE & Oct 1984 & Mar 2003 & $1365.4 \pm 0.6$ & Lee et al. (1987) \\
UARS & ACRIM-II & Oct 1990 & Nov 2001 & $1364.4 \pm 0.5$ & Pap et al. (1994) \\
SOHO & VIRGO & Feb 1996 & & $1365.7 \pm 0.6$ & Fröhlich et al. (1997) \\
ACRIM-sat & ACRIM-III & Apr 2000 & & $1366.2 \pm 0.7$ & Willson (2001) \\
SORCE & TIM & Mar 2003 & & $1360.9 \pm 0.4$ & Lawrence et al. (2000) \\
PICARD & PREMOS & Nov 2010 & & $1360.5 \pm 0.4$ & Schmutz et al. (2009) \\
\hline
\end{tabular}

(TRF) located at the Laboratory for Atmospheric and Space Physics (LASP) in Boulder, Colorado, USA (Fehlmann et al., 2012). The irradiance calibration is accurate to $330 \mathrm{ppm}$. PICARD/PREMOS agrees with SORCE/TIM to within $0.4 \mathrm{~W} \mathrm{~m}^{-2}$, with PICARD/PREMOS being lower (Schmutz et al., 2012). Thus, the new experiments are well within their common uncertainty range and the uncertainty difference between independent measurements of the TSI has decreased by a factor of ten.

The new experiments and the work carried out by the international community, which included realization of new facilities, ground-based tests, and collaborations aimed at identifying, quantifying, and verifying the causes of the discrepancy between the TIM and older TSI instruments, have ultimately led to the understanding of the instrument offsets. In particular, the characterisation of the SORCE/TIM, ACRIM/ACRIM III, and SOHO/VIRGO witness units at TRF, and the calibration of PICARD/PREMOS, led to better characterise instrumental effects and to resolve the source of the discrepancy among TSI observations (Kopp and Lean, 2011; Schmutz et al., 2012). Instruments such as PMO6 and ACRIM type having a view-limiting aperture in front and a smaller precision aperture that defines the irradiance area have a large amount of scattered light within the instrument. This additional light is not fully absorbed by the baffle system and produces scattered light contributing to extra power measured by the cavity. Scattered light was one of the potential systematic errors suspected by Butler et al. (2008). Subsequent ground testing involving the different instrument teams verified scattering as the primary cause of the discrepancy between the TIM measurements and the erroneously high values of other TSI instruments. Accordingly, new stray light corrections have recently been assigned to ACRIM/ACRIM-III (based on spare instruments) and its stray light contribution is indeed of the order as the observed differences. For SOHO/VIRGO the scattered light issue was not the reason for its discrepant reading. VIRGO is traceable to the World Radiometric Reference (WRR). New analyses, however, have revealed that the WRR has a systematic offset (Fehlmann et al., 2012). In particular, the WRR off- set produced approximately the same systematic shift as the scattering error. Thus, scattered light could be the reason of discrepant readings in both VIRGO and WRR instruments.

It is worth emphasizing that the key issue in irradiance measurements is the traceability of the instrument, which is necessary to meet metrological requirements. The various instruments have undergone increasingly precise post-launch corrections to meet such requirements; PICARD/PREMOS was the first to achieve complete traceability. However, there is now a consistent evaluation of the TSI from four instruments: SORCE/TIM, SOHO/VIRGO (corrected), ACRIM/ACRIM-III (corrected), and from PICARD/PREMOS. At the same time, TSI changes can be measured to much higher precision than absolute TSI values. On short timescales, relative measurements are accurate to a few ppm on a daily average. On longer timescales of years and tens of years, the stability of the measurements are much more difficult to evaluate. Claims of stabilities of less than 100 ppm over ten years (Fröhlich, 2009) are most likely too optimistic. A more realistic estimate comes from comparing independent composites that have been constructed. Over the time of the last solar cycle these agree to within about $0.2 \mathrm{~W} \mathrm{~m}^{-2}$ or about $20 \mathrm{ppm} \mathrm{yr}^{-1}$. For pre-1996 measurements even higher uncertainties for the systematic drifts have to be adopted. Despite these conservative assessments, the TSI time record is a factor of ten more accurate than any SSI observation.

\subsection{Discussion of SSI observations}

While the analysis of possible degradation trends for SORCE, SCIAMACHY, and other missions may help to resolve some of the differences in existing long-term timeseries of measurements, to advance our understanding of SSI variability new and improved, upgraded observations are required in the coming years. To this purpose, the next generation SIM instrument built for the NOAA/NASA Total Solar Irradiance Sensor (TSIS) mission (Cahalan et al., 2012) includes many design improvements for reducing noise and improving in-flight degradation tracking. The TSIS/SIM instrument is currently undergoing laboratory calibrations 
using radiometric technology similar to that employed to resolve the source of offsets among various TSI instruments (Kopp and Lean, 2011).

The TSIS mission might be launched in 2016. It is highly unlikely that TSIS/SIM and SORCE/SIM observations will overlap in time due to the expected lifetime of the SORCE batteries. Nevertheless, even without any overlap in time, the next generation measurements will help to better understand the performance of previous instruments and SSI solar cycle variation. In addition, several satellite missions dedicated to the observation of the Earth's atmosphere (GOME2, SBUV/2, OMPS (Ozone Mapping Profiler Suite)) will also provide information on part of the SSI spectra during the possible gap in observations from the instruments specifically designed to SSI measurements.

\section{Models of SSI variations}

Longer, uninterrupted, more stable and reliable observational time series are critical for understanding the origin of the differences between SSI measurements and improving our knowledge of SSI variations. However, the physics of the underlying processes also needs to be understood better, in order to facilitate the construction of more realistic models of SSI variations. Such models are particularly crucial since climate studies including stratospheric chemistry urgently need long and reliable SSI data sets for realistic simulations. Acquiring sufficiently long SSI time series is a long process and making them more reliable requires flying multiple new instruments, which will not happen for some time. Even when such time series do become available, they can only be extended into the past or future with the help of suitable models.

Although considerable progress has been made in modelling the TSI variability (e.g. Foukal and Lean, 1990; Chapman et al., 1996; Fröhlich and Lean, 1997; Fligge et al., 2000; Preminger et al., 2002; Ermolli et al., 2003; Krivova et al., 2003; Wenzler et al., 2004, 2005, 2006; Crouch et al., 2008; Bolduc et al., 2012; Ball et al., 2012), modelling the SSI is more difficult, leaving considerable room for improvement. Here we describe recent progress in SSI modelling. We discuss the mechanisms responsible for the irradiance variations (Sect. 3.1), with special emphasis on the possible differences in the spectral response of the irradiance to the various solar magnetic features. We then describe the basic principles and key components of the models and review five current SSI models (Sect. 3.2) that are available for climate studies and that were employed for our estimate of the atmospheric response to SSI solar cycle variations. Finally, we compare the models to each other, confront them with the available observational data presented in the previous section and discuss remaining uncertainties and open issues (Sect. 3.3).

\subsection{Mechanisms of irradiance variations}

Although various mechanisms have been proposed to explain the variation of solar irradiance, it is now accepted that observed variations in TSI (i.e. over the last 3.5 solar cycles) are predominantly caused by magnetic features on the solar surface. We cannot rule out that on longer timescales other mechanisms play a significant role, but this is beyond the scope of this paper.

Empirically it has been known for a long time that magnetic features on the solar surface are generally either dark (sunspots, pores) or bright (magnetic elements forming faculae and the network) when averaged over the solar disk. Two questions arise from this observation: why are some flux tubes (the theoretical concept used to describe faculae and sunspots) bright, while others are dark? What happens to the energy flux blocked by sunspots (or equivalently, where does the excess energy emitted by faculae come from)?

The strong magnetic field within both small and large magnetic flux tubes reduces the convective energy flux. The vertical radiative energy flux in the convection zone is comparatively small and cannot compensate a reduction in convective flux. This leads to a cooling of magnetic features.

The magnetic features are evacuated due to the large internal magnetic pressure and horizontal balance of total (i.e. gas plus magnetic) pressure. Hence, these evacuated magnetic structures are also heated by radiation flowing in from their dense and generally hot walls. This radiation efficiently heats features narrower than roughly $250 \mathrm{~km}$, making them brighter than the mainly field-free part of the photosphere, especially when seen near the limb where the bright walls are best visible (Spruit, 1976; Keller et al., 2004). For larger features, the radiation does not penetrate most of their volume (the horizontal photon mean free path is roughly $50-100 \mathrm{~km}$ ), so that features greater than roughly $400 \mathrm{~km}$ in diameter remain dark (pores and sunspots); cf. Grossmann-Doerth et al. (1994).

What happens with the energy that gets blocked by sunspots? According to Spruit (1982), this energy gets redistributed throughout the convection zone and is re-emitted again slowly over its Kelvin-Helmholtz timescale, which exceeds the lifetime of sunspots by orders of magnitude. Similarly, the excess radiation coming from small flux tubes (which act as leaks in the solar surface, since these evacuated features increase the solar surface area from which radiation can escape) is also taken from the heat stored inside the entire convection zone.

Total irradiance is simply the integral of SSI over wavelength. It is in the nature of integrals that quite different functions of wavelength, i.e. different SSI variations, can lead to the same variation in TSI. The differences in the relative change in irradiance at various wavelengths is given by three effects: (1) the relative sensitivity of the Planck function to temperature increases rapidly with decreasing wavelength. (2) Radiation at the various wavelengths is emitted 
at different heights in the solar atmosphere. This influences SSI because the contrast of magnetic features relative to their non-magnetic surroundings is height- and hence wavelengthdependent. (3) At very short and at very long wavelengths (EUV and radio), the radiation comes from the upper transition region and corona, where the brightest commonly found sources are complete loops rather than just the loop footpoints (the flux tubes) as at almost all wavelengths in between.

Point 3 refers to wavelengths we do not consider here because they interact mainly with the uppermost regions of the Earth's atmosphere (mesosphere and above) and hardly contribute to TSI at all. Point 2, however, is important, since in general the temperature in magnetic features drops more slowly with height in the solar photosphere and increases much more rapidly with height in the chromosphere than the solar average. This means that radiation emitted at higher levels in the solar atmosphere, i.e. in the UV and in the cores of spectral lines, displays larger changes. Together, points 1 and 2 favour the UV to exhibit larger variations than the visible and NIR. In addition to the fact that the continuum radiation comes from greater heights and that the Planck function shows a greater temperature sensitivity, the density of spectral lines per wavelength interval also increases very rapidly towards shorter wavelengths.

Now, can magnetic features be dark in the visible, but bright in the UV? This is in principle possible, if the magnetic feature is cool in the deep atmosphere, but hot in the upper photosphere. For instance, pores qualitatively show such a temperature profile, although there are no simultaneous high-resolution observations in the visible and the UV to decide if the temperature gradient is sufficiently extreme to produce such an effect. But pores are relatively short lived and too few in number. Hence, any long-lasting global dimming in the visible at times of high activity, such as that shown by the SORCE/SIM measurements, can only be produced by the small magnetic elements, specifically those in the network (these are much more numerous and more evenly distributed). However, such small-scale magnetic elements are unlikely candidates to produce a decrease in the visible irradiance along with increased UV irradiance. Firstly, magnetic elements in the network are bright even in the continuum and at disk centre (e.g. Kobel et al., 2011). Secondly, Röhrbein et al. (2011) have shown that the darker than average appearance of some magnetic elements is largely due to spatial smearing of the observations (although there may be some darkening due to the inhibition of convection around magnetic features). Thirdly, there are also many spectral lines in the visible, which brighten significantly in magnetic elements and counteract any darkening in the continuum. Finally and most importantly, magnetic elements near the limb are always rather bright, so that averaged over the solar disk small-scale magnetic features are expected to lead to a brightening. All this implies that the measurements by SORCE/SIM of a change in the irradiance at visible wave- lengths out-of-phase with TSI (Harder et al., 2009) are not compatible with the surface magnetic field as the source of the SSI variations in the visible. However, the SSI variations in the visible are an important contributor to TSI variations (Fig. 2) and the above incompatibility would be strongly contradicted by the result of Ball et al. (2012) that $92 \%$ of TSI variations are reproduced by the evolution of the magnetic field at the solar surface.

\subsection{Solar irradiance models}

It was noticed soon after the beginning of routine monitoring of TSI from space, that changes in TSI were closely related to the evolution of different brightness structures on the visible solar disc (Foukal and Vernazza, 1979; Willson et al., 1981; Oster et al., 1982; Eddy et al., 1982; Foukal and Lean, 1986). These brightness structures (such as sunspots, pores, faculae, plage and network) are manifestations of the solar magnetic field emerging at the Sun's surface. Thus their evolution in a global sense (and without looking too closely at the details of the temporal evolution) can be relatively well represented by different, typically disc-integrated, proxies of solar magnetic activity, such as the sunspot number or area, plage area, the solar radio flux at $10.7 \mathrm{~cm}$ (f10.7), the $\mathrm{Mg}$ II core-to-wing index, or the Ca II K line. This has widely been used in the oldest (but still widely deployed) irradiance models (e.g, Donnelly et al., 1982; Foukal and Lean, 1990; Chapman et al., 1996, 2012; Fröhlich and Lean, 1997; Fligge et al., 1998; Lean, 2000; Preminger et al., 2002; Jain and Hasan, 2004; Pagaran et al., 2009). In these models, the measured irradiance variations are fitted via a set of activity proxies through multiple regressions.

The success and the limitations of the regression methods in accounting for measured TSI variations (on timescales of days to years) gave a strong impetus to the development of more sophisticated and physics-based models. Such models consider contributions of different brightness structures to the irradiance change separately. Thus the solar energy output is the sum of the fluxes emerging from all the features observed on the solar visible surface (corresponding to the solar photosphere); the number and type of disk features accounted for depends on the model. Usually these models require two prime ingredients: (1) the surface area covered by each photospheric component as a function of time, and (2) the brightness of each component as a function of wavelength and often also of the position on the solar disc.

1. The surface area coverage by each photospheric component (i.e. sunspot umbrae and penumbrae, pores, faculae, plage, network, etc.) is derived from observations. These could be disc-integrated data (e.g. sunspot number, sunspot area, facular or plage area, $\mathrm{Mg}$ II index, cosmogenic isotope data, etc.; such as in the models by Fligge et al., 1998; Solanki and Unruh, 1998; Fligge and Solanki, 2000; Krivova et al., 2007, 2010; Shapiro et al., 2011; Vieira et al., 2011) or spatially resolved 
observations ( e.g. in the models by Fligge et al., 2000; Ermolli et al., 2003; Krivova et al., 2003; Fontenla et al., 2004, 2011; Fontenla and Harder, 2005; Wenzler et al., 2004, 2005, 2006; Unruh et al., 2008; Ball et al., 2011).

2. The brightness of individual photospheric components is calculated from semi-empirical models of the solar atmospheric structure using various radiative transfer codes, such as SRPM (Fontenla et al., 2011, and references therein), ATLAS9 (Kurucz, 1993) in SATIRE (Unruh et al., 1999; Fligge and Solanki, 2000; Krivova et al., 2003) or COSI (Haberreiter et al., 2008; Shapiro et al., 2010). Such computations are significantly complicated by the departures from the local thermodynamical equilibrium (LTE) conditions (Sect. 2.6 of Rutten, 2003) in the solar atmosphere, as well as by the temporal and spatial bifurcations of the temperature and density in the solar atmosphere (Carlsson and Stein, 1997; Uitenbroek and Criscuoli, 2011). High resolution 3-D models (e.g. Socas-Navarro, 2011) and 3-D MHD (magnetohydrodynamic) simulations (e.g. Vögler, 2005) of the solar atmosphere are gradually becoming available. However, current models cannot yet reproduce available observations over the entire spectrum (Afram et al., 2011). Thus, at present, semiempirical 1-D models of the solar atmosphere are a de facto standard for calculating irradiance variations. Although these models do not account for the spatial structure and temporal variability of the solar atmosphere, they can be adjusted to calculate the solar spectrum and its variability with high accuracy (Unruh et al., 1999; Fontenla et al., 2011, and references therein). These models do not necessarily catch the average properties of the inhomogeneous solar atmosphere (Loukitcheva et al., 2004; Uitenbroek and Criscuoli, 2011), but, if validated and constrained by the available measurements, they can be considered as a reliable and convenient semi-empirical tool for modelling SSI variability.

Presently, a wide range of solar atmospheric models with different degrees of complexity is available (e.g. Kurucz, 1993; Fontenla et al., 1999, 2009, 2011; Unruh et al., 1999; Penza et al., 2004; Uitenbroek, 2001; Kurucz, 2005; Avrett and Loeser, 2008; Shapiro et al., 2010), most of which go back to Vernazza et al. (1981).

The spectra of the quiet Sun calculated with three different models are shown in Fig. 6 (top panel). All plotted spectra are in reasonable agreement with each other, though models used in COSI and SATIRE are a bit closer to each other than to SRPM. The bottom panel in Fig. 6 shows the flux differences (i.e. the contrasts) between bright active components and the quiet Sun calculated with different models. The calculations were done assuming that the active regions cover the entire solar disk. To calculate the variability, the contrasts have to be weighted by the filling factors (surface area coverage), which are specific for every model. Thus, differ-
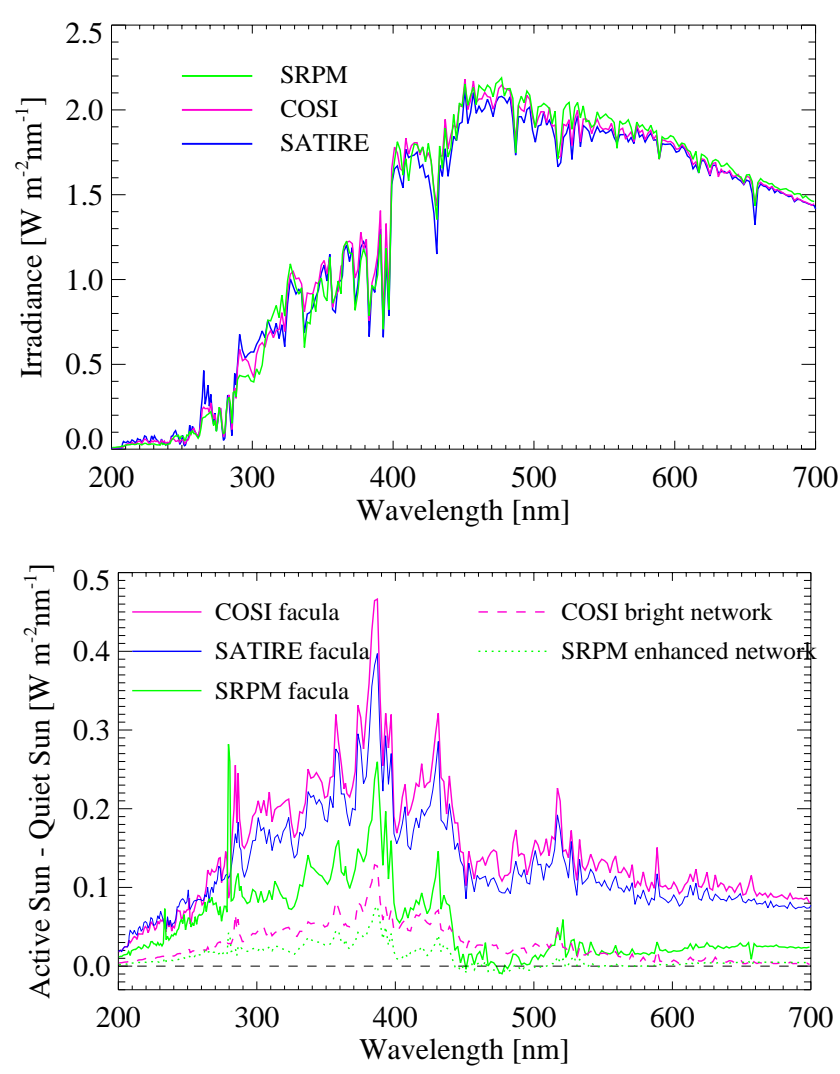

Fig. 6. Brightness of the quiet Sun (QS; top panel) and brightness contrasts of bright active components (bottom panel) in different models: SRPM (Fontenla et al., 2011, green lines), COSI (Shapiro et al., 2010, purple) and SATIRE (Unruh et al., 1999, blue). Note that contrasts depend on the position on the disc. Shown are averages over the entire disc under the assumption of a homogeneous spatial distribution of features.

ent magnitudes of the contrasts do not necessarily imply that the models produce different variability. At the same time, the spectral profile of the contrasts determines the dependence of the variability on the wavelength. Figure 6 shows that the (disk-integrated) spectral contrasts of facular, plage, and network regions calculated with SATIRE and COSI are very similar and positive over the whole spectral range. However, the SRPM contrasts are much lower in the visible compared to other models and are negative at some wavelengths. This is not very surprising, since the SRPM contrasts shown in Fig. 6 are derived from the most recent versions of the Fontenla et al. (1999) model family that were subsequently revised to account for some observations of the solar atmosphere, as further detailed below.

The advantage of employing radiances computed from the semi-empirical model atmospheres is that they allow computations of solar irradiance at different wavelength (i.e. spectral irradiance). This is not straightforward with the regression models, since in this case the regression coefficients 


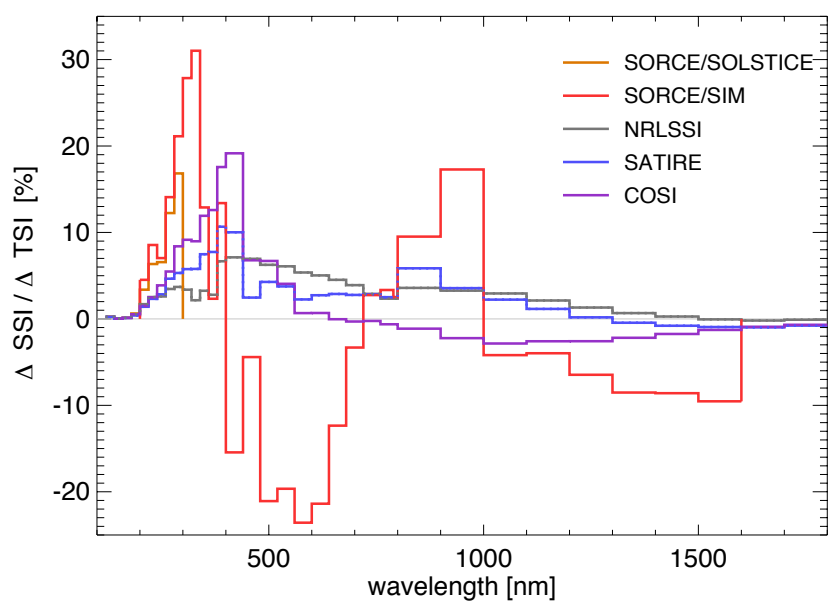

Fig. 7. Ratio of SSI variability to TSI variability between $200 \mathrm{~nm}$ and $1800 \mathrm{~nm}$ in bins of $20 \mathrm{~nm}$ below $400 \mathrm{~nm}, 40 \mathrm{~nm}$ in the range $400-800 \mathrm{~nm}$, and $100 \mathrm{~nm}$ in the IR above $800 \mathrm{~nm}$. Shown are SORCE/SOLSTICE (orange) and SORCE/SIM (red) measurements between 2004 and 2008, as well as the NRLSSI (grey), SATIRE-S (blue) and COSI (purple) models between the maximum and minimum of cycle 23 .

need to be estimated from observations or, alternatively, irradiance changes at individual wavelengths need to be somehow scaled from the TSI changes or changes in the irradiance at some other (known) wavelength. This latter technique is also partly used in the UV by the NRLSSI and SATIRE models that are described below.

\subsubsection{NRLSSI}

The Naval Research Laboratory Solar Spectral Irradiance ${ }^{2}$ (NRLSSI; Lean et al., 1997; Lean, 2000) uses the photospheric sunspot index derived from sunspot area records to describe the evolution of sunspots in time, and $\mathrm{Mg}$ II, CaII, and f10.7 disk-integrated indices to represent facular brightening.

Below $400 \mathrm{~nm}$, the spectral irradiances are derived from UARS/SOLSTICE observations through a multiple regression analysis with respect to a (SOLSTICE) reference spectrum. The regression analysis includes a facular brightening and a sunspot blocking component (see Lean et al., 1997; Lean, 2000 for more detail). It is known that the long-term stability of the UARS spectral instruments (both SOLSTICE and SUSIM) was not sufficient to trace solar cycle variability above roughly $220 \mathrm{~nm}$ (Woods et al., 1996). Therefore the coefficients are derived from the rotational variability so as to avoid any long-term instrumental effects. This approach thus assumes that spectral irradiance changes show the same linear scaling with a given proxy on rotational as well as cyclical timescales.

\footnotetext{
${ }^{2} \mathrm{http}: / /$ lasp.colorado.edu/lisird/nrlssi/
}

Above $400 \mathrm{~nm}$, the facular and sunspot contrasts are largely based on the contrasts presented in Solanki and Unruh (1998). They have been scaled to ensure that the overall (wavelength-integrated) solar cycle change due to sunspots (viz. faculae) agrees with the bolometric value for the sunspot blocking (viz. facular brightening) derived from TSI modelling (see, e.g. Fröhlich and Lean, 2004).

The quiet-Sun spectrum in NRLSSI is effectively a composite of UARS/SOLSTICE observations (below $400 \mathrm{~nm}$ ), SOLSPEC (Thuillier et al., 1998), and the model by Kurucz (1991). This composite has been scaled so that its integrated flux corresponds to a TSI value of $1365.5 \mathrm{~W} \mathrm{~m}^{-2}$.

Compared to the SATIRE and COSI models (described below), the NRLSSI model shows lower variations on solarcycle and longer timescales between 250 and $400 \mathrm{~nm}$ (Figs. 2 and 7). This is mainly because the regression coefficients are derived from rotational variability only. Below $250 \mathrm{~nm}$ (Figs. 7 and 8) and in the visible at $400-700 \mathrm{~nm}$ (Figs. 2 and 7) the NRLSSI, COSI, and SATIRE models agree well with each other.

In the visible and IR range, the facular contrasts in the NRLSSI model rely on the facular model atmosphere by Solanki and Unruh (1998), which is an earlier version of the model by Unruh et al. (1999) currently used in SATIRE. Thus although the NRLSSI model does show a weak out-of-phase variability in the IR around $1600 \mathrm{~nm}$ (Fig. 7), integrated over the range $1000-2430 \mathrm{~nm}$ (Fig. 2) the modelled irradiance is in phase with the solar cycle. The SATIRE, COSI, and OAR models all display reversed variability in this range. Such a reversed behaviour is also suggested by the SORCE/SIM data, although the magnitude of the measured SIM variability is much stronger than in the models.

Integrated over all wavelengths, the NRLSSI irradiance (i.e. the TSI) is in good agreement with the measurements, although it does not quite reproduce the comparatively low TSI level during the last minimum in 2009 (Kopp and Lean, 2011), as indicated by the PMOD composite and also independently found by Ball et al. (2012) with the SATIRE model. This is because the Mg II index employed to describe the evolution of the bright component with time does not follow exactly the shape of the TSI variation over the last cycle (although the differences appear to be within the long-term measurement uncertainties; M. Snow, personal communication, 2012).

\subsubsection{SATIRE-S}

SATIRE-S ${ }^{3}$ (Spectral And Total Irradiance REconstructions for the Satellite era) belongs to the class of models using semi-empirical model atmospheres to calculate brightnesses of different surface features and full-disc solar images to describe the surface area coverage by these components at a given time. The full-disc data used in SATIRE are

\footnotetext{
${ }^{3}$ http://www.mps.mpg.de/projects/sun-climate/data.html
} 

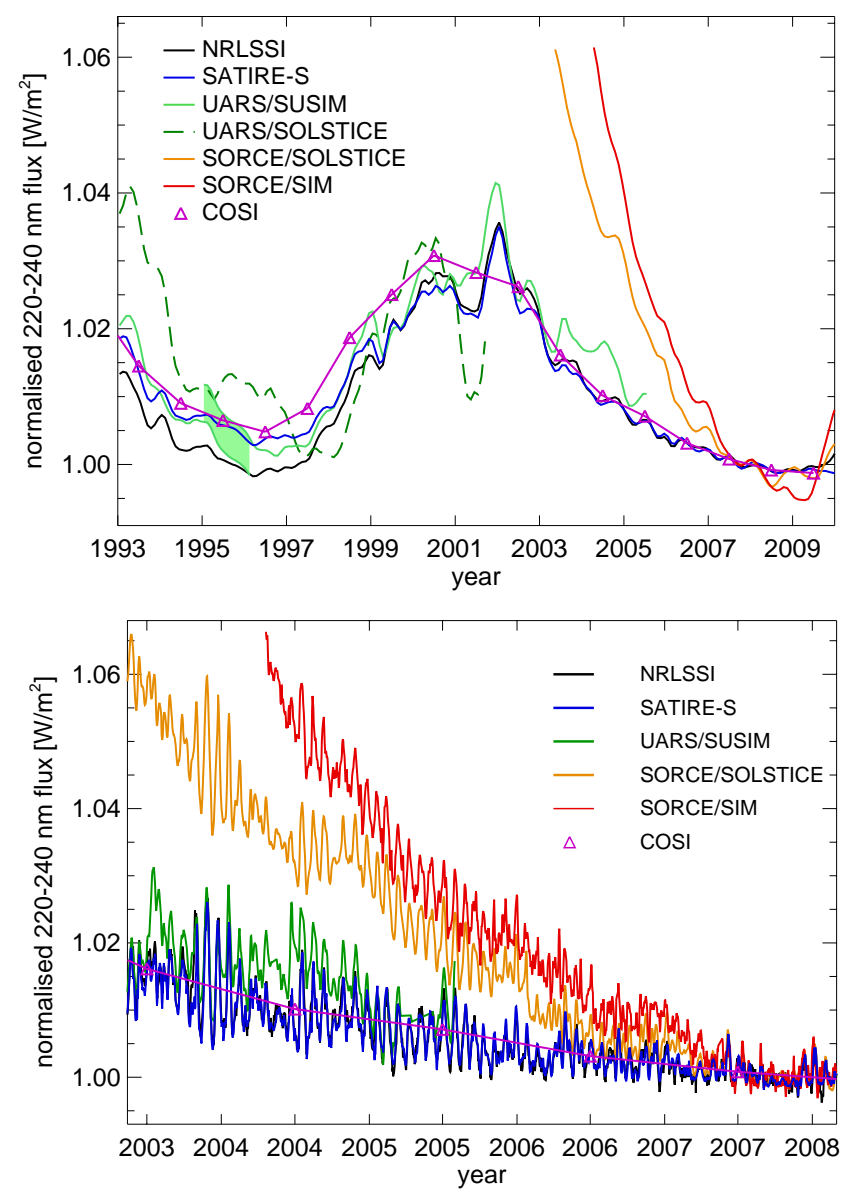

Fig. 8. Solar UV irradiance between 220 and $240 \mathrm{~nm}$ calculated with NRLSSI (black), SATIRE-S (blue) and COSI (magenta), and measured with UARS/SUSIM (darker green), UARS/SOLSTICE (light green), SORCE/SOLSTICE (orange) and SORCE/SIM (red). The pale green shading in the upper panel marks the period when the sensitivity of the UARS/SUSIM instrument (and thus the flux) changed, so that a shift was applied to the data before that (see Krivova et al., 2006, 2009 for details). Top panel shows 3-month smoothed values over the period 1993-2009, while the bottom panel is limited to the period when SORCE was in operation, i.e. after 2003, and shows daily values, except for the COSI model, for which only yearly averages are available. All quantities are normalised to the corresponding mean values during the last activity minimum (2009).

magnetograms and continuum images. So far, ground-based KP/NSO (Kitt Peak National Solar Observatory; 1974-2003) and space-based SOHO/MDI (Michelson Doppler Interferometer; 1996-2010) and SDO/HMI (Helioseismic and Magnetic Imager; since 2010) data have been employed. The following model atmospheres are used: the quiet Sun model by Kurucz (1991) with an effective temperature of 5777 K, similar but cooler model atmospheres for umbra and penumbra, and model P of Fontenla et al. (1999) slightly modified by Unruh et al. (1999) to achieve better agreement with obser- vations in the visible and near-UV. Since the ATLAS9 code uses the LTE approximation, which is known to fail in the UV, fluxes below $270 \mathrm{~nm}$ are re-scaled using UARS/SUSIM observations (Krivova et al., 2006).

The SATIRE-S modelled variability was found to be in very good agreement, on both rotational and cyclic timescales, with the PMOD TSI composite (Wenzler et al., 2009; Ball et al., 2012), SORCE/TIM TSI measurements (Ball et al., 2011, 2012), UARS/SUSIM spectral irradiance (Krivova et al., 2006, 2009; Unruh et al., 2012), as well as with UARS/SUSIM and UARS/SOLSTICE Ly- $\alpha$ measurements (Figs. 2 and 8). On rotational times scales, good agreement is also found between the SSI provided by SATIRE$\mathrm{S}$ and the SORCE/SIM and SORCE/SOLSTICE measurements (Unruh et al., 2008, 2012; Ball et al., 2011, see also bottom panel in Fig. 8) and between SATIRE-S and SOHO/VIRGO observations in three spectral channels in the near-UV, visible, and near-IR (Krivova et al., 2003). Due to strong sensitivity trends, VIRGO spectral data could not be used on longer timescales. On time scales longer than a few months, the SATIRE-S trends, however, diverge significantly from those shown by SORCE/SIM and SORCE/SOLSTICE: the change in the UV is significantly weaker in SATIRE-S, and the inverse solar cycle change in the visible is not reproduced (Figs. 7 and 8; Ball et al., 2011; Unruh et al., 2012). Interestingly, Ball et al. (2011) have shown that when integrated over the spectral range of SIM (200-1630 nm) and corrected for the missing UV and IR wavelengths, SATIRES still reproduces over $94 \%$ of TSI fluctuations measured by SORCE/TIM. The trends of smoothed data agree to over $99 \%$. At the same time, wavelength-integrated SORCE/SIM data show different trends (only about $60 \%$ of the TIM changes are reproduced over the SORCE/SIM life time), though the uncertainties in the integrated SORCE/SIM data are quite large (see Ball et al., 2011).

\subsubsection{SRPM}

The Solar Radiation Physical Modelling ${ }^{4}$ (SRPM) is a set of tools used to construct semi-empirical models of the solar atmosphere and to derive solar radiance (or emitted intensity) and high-resolution irradiance spectra of the Sun. SRPM covers all levels of the solar atmosphere from the photosphere to the corona and takes, for most species, NLTE conditions into account. The calculations include a large number of atomic levels and lines, as well as molecular lines and molecular photo-dissociation opacities. Seven components have been used in SRPM, until recently, corresponding to different features that can be identified on the Sun at a medium-resolution of $\approx 2$ arc sec: quiet-Sun inter-network, quiet-Sun network lane, enhanced network, plage (that is not facula), facula (i.e. very bright plage), sunspot umbra and sunspot penumbra. The atmospheric models adopted for

\footnotetext{
${ }^{4}$ http://www.digidyna.com/Results2010/
} 
the calculations of each of these features are presented in Fontenla et al. (2009). These models go back to the ones of Fontenla et al. (1999, and references therein), which have been revised in subsequent studies of Fontenla et al. (2004, 2006, 2009) to account for observational results and for extension of the atmospheric layers. The observational results include e.g. the negative contrast measurements on facular regions at some wavelengths (Foukal et al., 1990; Topka et al., 1992; Wang et al., 1998). Recently, further modifications were introduced into the plage models to take SORCE data into account (see below). In addition, two new components and corresponding model atmospheres, namely dark quiet-Sun inter-network and hot facula, were added but not yet integrated into solar irradiance calculations presented in Fontenla et al. (2011). For irradiance calculations, the distribution of the atmospheric components over the solar disc at a given time is derived from full-disc solar filtergrams; the ones employed so far include observations from the Precision Solar Photometric Telescopes (PSPT; Coulter and Kuhn, 1994) at the INAF Osservatorio Astronomico di Roma and at Mauna Loa, as well as Meudon photographic images.

Fontenla et al. (2011) presented SRPM-based calculations of daily SSI at $1 \mathrm{~nm}$ resolution for the period 2000-2009 using observations carried out with the PSPT telescope in Rome. The atmospheric model set used in Fontenla et al. (2011) is essentially that of Fontenla et al. (2009) with a few modifications, e.g. to photospheric layers of the plage models (see Fontenla et al., 2011). These calculations produce a decrease in irradiance at wavelengths below $\approx 400 \mathrm{~nm}$ during the declining phase of solar activity from 2002 to 2009 , and a flux increase at longer wavelengths, similarly to the behaviour seen in the SORCE/SIM observations (Harder et al., 2009). The UV variability derived from the model is, however, significantly weaker than what is measured by SORCE/SOLSTICE. Moreover, the observed changes of TSI on timescales longer than the solar rotation are not captured by the model. Fontenla et al. (2011) argue that a possible reason for this mismatch could be that the current set of the employed atmospheric models is still missing some components.

\subsubsection{COSI}

The COde for Solar Irradiance ${ }^{5}$ (COSI) calculates synthetic solar spectra for different components of the solar atmosphere. COSI returns the most important UV lines including the degree of ionisation of the elements under NLTE. The NLTE opacity distribution functions (Hubeny and Lanz, 1995), implemented in COSI by Haberreiter et al. (2008), indirectly account for the NLTE effects in several millions of lines. Shapiro et al. (2010) showed that NLTE effects influence the concentration of the negative ion of hydrogen, which results in an approximately $10 \%$ change of the continuum

\footnotetext{
${ }^{5} \mathrm{ftp}: / / \mathrm{ftp} . \mathrm{pmodwrc} . \mathrm{ch} / \mathrm{pub} / \mathrm{Sasha} /$
}

level in the visible spectrum. The contrasts between active regions and the quiet Sun are also affected by the NLTE effects. This, however, does not imply that only NLTE codes are capable of reproducing the visible solar spectrum as the NLTE effects can be imitated by a slight readjustment of the atmosphere structure (see, e.g. Rutten and Kostik, 1982; Shchukina and Trujillo Bueno, 2001). The spectrum of the quiet Sun calculated with COSI is in good agreement with the solar spectrum measured by SOLSTICE (up to $320 \mathrm{~nm}$ ) and SIM (above $320 \mathrm{~nm}$ ) onboard the SORCE satellite during the 2008 solar minimum, and with the SOLSPEC measurements during the ATLAS 3 mission in 1994 (Thuillier et al., 2011). Shapiro et al. (2013a) showed that COSI can accurately reproduce the center-to-limb variation of solar brightness in the Herzberg continuum retrieved from the analysis of solar eclipses observed by LYRA/PROBA2 (Large Yield RAdiometer/PRoject for On-Board Autonomy).

Solar spectral irradiance variations can be modelled from COSI spectra by weighting them with filling factors of surface magnetic features (e.g. derived from the magnetograms as in the SATIRE model, cf. Haberreiter et al. (2005), or from the PSPT images as in SRPM). In the current version of the model, this has been done employing sunspot and ${ }^{10} \mathrm{Be}$ data (Shapiro et al., 2011), and PSPT images (Shapiro et al., 2012).

The variability so derived with COSI agrees well with SATIRE-S and NRLSSI in the Herzberg continuum spectral range (Figs. 7 and 8 ) and in the visible (Figs. 2 and 7). In the near-IR, at $700-1000 \mathrm{~nm}$, COSI shows a weak inverse solar cycle variability (Figs. 2 and 7). This is probably an artefact of the model, as Shapiro et al. (2010) calculated active-region contrasts using the model atmospheres of Fontenla et al. (1999), which do not distinguish between umbra and penumbra. Besides, they employed a single model for both bright plage and plage. These assumptions affect the position of the inversion point; the point where the influence of sunspots starts to outweigh the bright components. To account for this, Shapiro et al. (2012) decreased plage and sunspot contrasts with respect to the quiet Sun. A more accurate approach is under development.

\subsubsection{OAR}

Full-disc observations carried out with the PSPT telescope in Rome (Ermolli et al., 1998, 2007, 2010) are also employed in the OAR (Osservatorio Astronomico di Roma) model of solar irradiance variations. Earlier results obtained with both a regression method and a semi-empirical model were presented by, e.g. Penza et al. (2003, 2006), Domingo et al. (2009) and Ermolli et al. (2011). A new semi-empirical model, briefly outlined below, is currently under development (Ermolli et al., 2012).

Images taken between September 1997 and January 2012 have been processed and segmented to identify different features on the solar disc as described by Ermolli et al. 
(2010). The seven classes of atmospheric features proposed by Fontenla et al. (2009) are considered. The brightness spectrum of each feature is computed using the semi-empirical atmospheric models of Fontenla et al. (2009) through the RH synthesis code (Uitenbroek, 2002). Thus the set of components in the OAR model is essentially the same as used by Fontenla et al. (2011), though their atmospheric structure is based on Fontenla et al. (2009), i.e. before the most recent modifications applied by Fontenla et al. (2011).

The SSI variation calculated with the OAR model in the visible (400-691 nm) over the period 2000-2012 shows a trend that is opposite to that measured by SORCE/SIM and obtained with SRPM calculations. The variability in the UV (200-400 nm) estimated with the model is significantly weaker than that depicted by the SORCE measurements. However, the TSI variations calculated with the model agree well with the PMOD composite and SORCE/TIM measurements on both rotational and cyclic timescales (Ermolli et al., 2012), thus suggesting that there is no need for additional components to reproduce the observed TSI changes.

\subsection{Discussion of SSI model results}

There has been steady progress in modelling SSI variations, and a number of models are now available that can be used as input for climate studies. However, the main uncertainty in the models concerns the wavelength range $220-400 \mathrm{~nm}$, which is of particular interest for climate studies. Of the five models discussed in this section, specifically NRLSSI, SATIRE-S, COSI, SRPM and OAR, only one (SRPM) shows a behaviour of the UV and visible irradiance qualitatively resembling that of the recent SORCE/SIM measurements. However, it should be noted that the integral of the SSI computed with this model over the entire spectral range (i.e. the TSI) does not reproduce the measured cyclical TSI changes. None of the other four models, which are in closer agreement with each other, reproduces the behaviour of the UV and visible irradiance observed by SORCE/SIM (Figs. 2, 7 and 8). These models are nevertheless all in good or fair agreement with earlier UARS UV observations, TSI measurements, and the model based on SCIAMACHY data (e.g. Krivova et al., 2006, 2009; Pagaran et al., 2009; Morrill et al., 2011a; Shapiro et al., 2011; Unruh et al., 2012; Lean and DeLand, 2012; Ball et al., 2012). These models also agree with SORCE data on rotational times scales (e.g. Unruh et al., 2008; Ball et al., 2011; Lean and DeLand, 2012, see also bottom panel in Fig. 8). Note that good agreement on rotational timescales was also found by DeLand and Cebula (2012) between SORCE and other spectral observations.

When comparing various models and data to each other, however, one important issue often remains unacknowledged, namely the true uncertainties in the measurements, in particular on timescales of years and longer. As an example, we compare measured and modelled variability at 220 $240 \mathrm{~nm}$ (Fig. 8). Over the period 2004-2008, the three mod- els shown in this figure (NRLSSI, SATIRE-S and COSI) suggest a decrease in the integrated 220-240 nm flux of about $1 \%$. The fluxes measured by the SORCE/SOLSTICE and SORCE/SIM instruments in this range decreased over the same period by roughly $4 \%$ and $7 \%$, respectively. In the 220-240 nm region, the accuracy of SORCE/SOLSTICE is considered to be better than that of SIM (Harder et al., 2010). Currently, SOLSTICE long-term stability in this spectral range is estimated to be about $1 \% \mathrm{yr}^{-1}$ (Snow et al., 2005). Thus the observed trend for SORCE/SOLSTICE ( $4 \%$ over $5 \mathrm{yr}$ ) and its difference with the models ( $3 \%$ over $5 \mathrm{yr}$ ), in fact, lies within the long-term instrumental uncertainty. The difference between the trends for SORCE/SIM and the models (about $6 \%$ ) is just outside the value of $5 \%$ over $5 \mathrm{yr}$, assuming that the SIM long-term stability is comparable to that of SOLSTICE. Although the SIM uncertainty stated in Merkel et al. (2011) is lower, this assumption seems to be a reasonable working hypothesis for our study, because it is known that at 220-240 SIM has poorer precision than SORCE/SOLSTICE (J. Harder, personal communication, 2012). It is worth mentioning that new assessments of the SORCE instrument degradation might imply a revision of results derived from several studies, including this one, which however is mainly intended to discuss the data and models available to date for the evaluation of the atmospheric response to SSI variations.

Despite showing much lower variability than SORCE/SIM in the UV, the models also display considerable differences between each other in the range $250-400 \mathrm{~nm}$ (up to a factor of three, e.g. between NRLSSI and COSI; Figs. 2 and 7). As a consequence of the low response of UARS/SOLSTICE to long-term variability above $300 \mathrm{~nm}$ and the use of rotational variability to estimate the regression coefficients, it is likely that NRLSSI underestimates the changes in this range (J. Lean, personal communication, 2012), and can thus be considered as the lower limit. All other models rely on semiempirical model atmospheres in this range, which also need further tests at all wavelengths. The differences between the models in the IR are mainly due to the lack of reliable observations of contrasts of different (solar) atmospheric components and related uncertainties in the corresponding model atmospheres. In the near-IR $(700-1000 \mathrm{~nm})$, all models qualitatively agree, except COSI. However, as discussed earlier, the inverse variability shown by COSI in this range is believed to be an artefact of the atmospheric components and models adopted for the irradiance calculations.

The variability of the spectral irradiance on timescales longer than the solar cycle is beyond the scope of this paper. We note, however, that uncertainties in the long-term SSI reconstructions are essentially the same as the ones discussed in this section convolved with the uncertainty in the magnitude of the secular change in irradiance (see, e.g. Krivova and Solanki, 2013; Schmidt et al., 2012; Solanki et al., 2013, and references therein). 


\section{Climate impact of SSI measurements}

The SSI data derived from recent solar observations and model calculations presented in the previous sections are employed here to discuss the impact of SSI variations on the Earth's atmosphere and climate. In particular, we present the motivation for simulations of the response of the Earth's atmosphere to variations induced by SSI changes (Sect. 4.1). We then discuss the solar induced differences in atmospheric heating rates, ozone variability, temperature, and atmospheric circulation (Sect. 4.2). These differences are investigated in recent simulations using atmospheric models with standard solar forcing from the NRLSSI model (e.g. SPARC-CCMVal, 2010; Taylor et al., 2012, for CMIP5 simulations). Additionally, a comparison to recent atmospheric model simulations using the SORCE measurements as solar forcing (e.g. Haigh et al., 2010; Merkel et al., 2011; Oberländer et al., 2012) is provided. As shown and discussed in the previous sections, the NRLSSI model output and the SORCE measurements represent the lower and the upper boundaries of current understanding of SSI solar-cycle variability. Here we provide an estimate of the uncertainty in the magnitude of solar induced atmospheric changes in CCMs by comparing the impact from the two extremes in the current evaluation of SSI variability (Sect. 4.3).

\subsection{Climate modelling}

Today, the most advanced tools available for climate simulations are 3-dimensional general circulation models (GCMs) that numerically simulate the general circulation of the atmosphere and/or the ocean based on well-established physical principles. Most climate models that are utilised for future climate predictions in the 4th IPCC report (Solomon et al., 2007) are coupled atmosphere-ocean models reaching up to the middle stratosphere $(32 \mathrm{~km})$, whereas CCMs that are used for future predictions of the stratospheric ozone layer in the WMO (World Meteorological Organization) report (WMO, 2011) include interactive stratospheric chemistry and reach up to the lower mesosphere or above $(80 \mathrm{~km})$. The main external driving force for all climate models is the incoming solar flux at the top of the atmosphere (TOA). Currently there are two mechanisms for solar radiation influence on climate, the so-called "top-down" UV effect (Kodera and Kuroda, 2002) and the "bottom-up" TSI effect (van Loon et al., 2007).

As discussed in the previous sections, even though TSI varies only by about $0.1 \%$ over the solar cycle, larger variations of several percent occur in the UV part of the spectrum, including the ozone absorption bands between 200 and $400 \mathrm{~nm}$ that are responsible for the SW heating of the stratosphere and are important for photochemical processes (e.g. Haigh, 1994). The cyclic variation of the incoming solar irradiance at short wavelengths leads to statistically significant ozone, temperature, and zonal wind solar signals in the stratosphere (Austin et al., 2008; Gray et al., 2010). These solar induced circulation changes in the stratosphere can induce noticeable decadal climate changes in the lower atmosphere and at the surface (e.g. Haigh, 1999; Kodera, 2002; Matthes et al., 2006; Ineson et al., 2011; Matthes, 2011). In order to account for this so-called "top-down" stratospheric UV mechanism, the radiation code in climate models has to account for spectrally resolved irradiance changes. The first climate models focused on tropospheric climate, thus, solar forcing was represented by TSI only. As stratospheric changes played a minor role for climate predictions in the past, climate models did not take into account stratospheric processes and, in particular, ozone changes due to solar UV absorption. Hence, most of the SW radiation codes developed for use in GCMs did not consider solar irradiance for wavelengths shorter than $250 \mathrm{~nm}$ and employed parameterization using TSI as input. Solar fluxes and heating rates were subsequently calculated in one or two SW absorption bands from the top of the atmosphere to the surface. In contrast, middle-atmosphere models use SW radiation codes specifically designed for simulations of the upper atmosphere. They, therefore, cover a broader spectral range and include more than two spectral bands in the UV and visible.

Studies on the performance of SW radiation codes with different spectral resolution showed that the observed solar temperature signal in the stratosphere can only be reproduced in models that allow for the effects of spectral variations between solar minimum and maximum (Egorova et al., 2004; Nissen et al., 2007). In a recent paper, Forster et al. (2011) examined in detail the sensitivity of a number of CCM SW radiation codes to changes in solar irradiance and ozone as well as the ability of the models to reproduce the $11 \mathrm{yr}$ radiative solar signal using the NRLSSI data. In their study (SPARCCCMVal, 2010), the strongest solar temperature signal was found to be in the tropical upper stratosphere/lower mesosphere, indicating that the direct mechanism of heating by absorption of enhanced UV radiation at solar maximum is well captured by the models that employ spectrally resolved SW radiation schemes. Models that do not account for SSI variations and only consider changes in spectrally integrated TSI cannot properly simulate solar induced variations in stratospheric temperature (Forster et al., 2011).

Today, stratospheric processes are gaining a lot of interest due to their importance for climate. Not only the effect of ozone recovery and its relationship to climate but also stratosphere-troposphere dynamical coupling and its role for predictability from days to decades have been recognised as important issues for future climate studies (e.g. Baldwin et al., 2007; Gerber et al., 2010). Therefore, a better representation of the stratosphere including improved representation of SW heating processes as well as dynamical coupling with the troposphere in global climate models is critically important. 


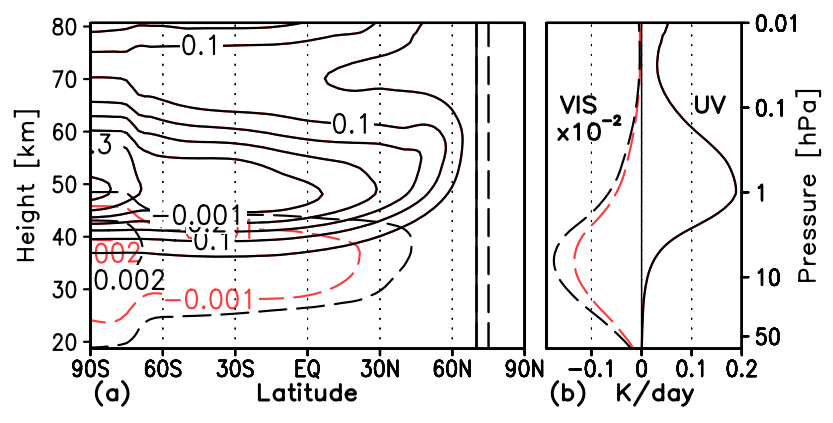

Fig. 9. Updated from Oberländer et al. (2012) (a) difference in zonal mean solar heating rates between 2004 and 2007 in $\mathrm{Kday}^{-1}$ from the SIM data, and (b) difference in the global mean 2004-2007 solar heating rate signal between SORCE and NRLSSI data. Solid lines denote the UV spectral range, and dashed lines denote the VIS spectral range. Red dashed lines in panels (a) and (b) denote the same differences in zonal and global mean, as calculated and presented in Fig. 3b, c in Oberländer et al. (2012); black dashed lines indicate the enhanced solar signal in the VIS range, when extending the spectral resolution of the FUBRad shortwave radiation code from one to six bands in the Chappuis bands. The simulations were performed for perpetual January conditions.

\subsection{Impact of SSI variability in climate models}

The uncertainty of SSI variations in recent observations and models has significant influence on simulations of the climate system, since the response of the atmosphere strongly depends on the spectral distribution of the solar irradiance. The effects for middle atmosphere heating, ozone chemistry and middle-atmospheric temperatures are examined in the following.

\subsubsection{Effects on atmospheric heating and ozone chemistry}

As solar radiation is the primary source of energy that drives atmospheric as well as oceanic circulation, accurate representation of solar irradiance is of paramount importance for the simulation of the atmospheric temperature, composition, and dynamics in climate models. The variability of the solar spectrum in time, and in particular over the solar cycle is necessary for the assessment of solar influence on climate. The amplitude of the simulated solar signal depends on the spectral solar fluxes prescribed at the TOA. Differences in the TOA solar irradiance spectrum result in large changes in the heating rates calculated by SW radiation schemes or radiative transfer models, as has been shown by Zhong et al. (2008) with differences of up to $\approx 1.1 \mathrm{Kday}^{-1}$ in midlatitude summer. Recently, Oberländer et al. (2012) examined the impact of a number of different estimates of prescribed TOA solar fluxes on the solar response in a GCM that includes a radiation scheme with enhanced spectral resolution (Nissen et al., 2007) and is therefore able to accurately represent the solar signal induced changes. They used the NRLSSI, the
SATIRE, and the SCIAMACHY solar flux input data sets, and compared their effects on SW heating rates over the $11 \mathrm{yr}$ solar cycle using offline calculations. They also calculated the corresponding temperature response from perpetual January GCM simulations with prescribed ozone concentrations.

The comparison revealed clear differences in SW heating rates for the solar minimum of cycle 22 (September 1986). The simulations forced with the NRLSSI reconstructions show the smallest solar heating rates. The use of the SATIRE reconstructions leads to stronger solar heating of up to $5 \%$ in the middle and upper stratosphere. The SCIAMACHY observations slightly enhance the solar heating in the mesosphere, with differences arising from the stronger solar fluxes in the Huggins bands of the SATIRE model and enhanced fluxes in the Hartley bands of the SCIAMACHY data set. Using SORCE (SIM and SOLSTICE for wavelengths below 210 $\mathrm{nm})$ measurements over the period May 2004 to November 2007 reveals larger changes in solar heating rates and the resulting temperatures in comparison to the NRLSSI data. The lower irradiance in the visible range at higher solar activity than at minimum activity in the SORCE/SIM data does not lead to a decrease in total radiative heating.

The spectral resolution of the SW radiation scheme of EMAC-FUB (European Multisensor Airborne Campaign/Freie Universität Berlin) has recently been extended towards a more detailed representation of the Chappuis bands, and an update of Oberländer et al. (2012), performed with the extended spectral resolution for solar minimum conditions in November 2007, separated for the UV and VIS spectral changes is presented in Fig. 9. The enhanced UV irradiance in 2004 (compared to 2007) in the SORCE data leads to higher SW heating rates while lower heating rates are simulated in the visible spectral range (Fig. 9a). The SW heating rate change from 2004 to 2007 is stronger when the model is forced with the SORCE than with the NRLSSI data by $0.18 \mathrm{Kday}^{-1}$ in the global mean (Fig. 9b). An enhanced sensitivity to changes in the Chappuis bands is found, a result which illustrates better the heating rate changes in the UV, visible, and NIR spectral regions from using the SORCE data.

The different magnitude and spectral composition of the SSI changes leads not only to a substantial alteration of heating rates considered above but also affects photolysis rates that drive atmospheric chemistry and regulate the atmospheric ozone distribution in the middle atmosphere (e.g. Brasseur and Solomon, 2005). The global ozone abundance is maintained by ozone production, destruction, and transport by air motions. However, in the tropical stratosphere above $\sim 30 \mathrm{~km}$ the ozone concentration depends primarily on photochemical processes, with oxygen photolysis playing a crucial role in atmospheric chemistry. Therefore, the spectral composition and the magnitude of the SSI changes are of critical importance, since they define not only the magnitude but also the sign of the direct ozone response (e.g. Rozanov 
et al., 2002). The solar induced net effect of ozone in the stratosphere depends on the competition between ozone production due to oxygen photolysis in the Herzberg continuum (185-242 nm) and ozone destruction caused by the ozone photolysis in the Hartley band (between 200 and $300 \mathrm{~nm}$ in the UV).

\subsubsection{Effects on ozone and temperature from atmospheric model simulations}

The effects of different SSI estimates on stratospheric ozone as deduced by CCMs or 2-D radiative-photochemical model simulations have been recently reported in a number of papers, which are discussed and compared to each other in the following. In particular, a number of analyses focused on the differences in atmospheric response between simulations using the SORCE and the NRLSSI model results. The NRLSSI is the data set widely used for climate modelling purposes, including the SPARC CCMVal (ChemistryClimate Model Validation) and the CMIP5 simulations for the upcoming IPCC report (Taylor et al., 2012). Cahalan et al. (2010) and Haigh et al. (2010) published first the important implications of the SORCE data for middle-atmosphere heating and therefore temperatures. Using simulations from a 2D radiative-photochemical model, Haigh et al. (2010) also presented effects on ozone. A number of other studies using 3-D chemistry-climate models followed (Merkel et al., 2011; Ineson et al., 2011; Matthes, 2011; Oberländer et al., 2012; Swartz et al., 2012). To better understand the atmospheric sensitivity to the range of SSI estimates discussed above, these recently published model experiments using the NRLSSI and the SORCE data are compared to each other in Figs. 10 and 11 with respect to the solar signal in SW heating rates, temperatures, and ozone. Additionally, results from three CMIP5 model experiments using the NRLSSI data set are shown. Table 2 presents an overview of the models and their experimental designs. Details on the spectral solar forcing used in each simulation are given in Table 3. The purpose is to provide the reader with an initial comparison of the impacts of two SSI data sets, which represent the lower and upper boundary of SSI variations. One has to keep in mind, though, that all models used slightly different experimental set-ups (including slightly different spectral ranges for the SORCE data) and therefore an exact comparison between them as well as to observations awaits common coordinated experiments. Moreover, we note that the solar spectral forcing from the SORCE satellite used by all the models is derived from a relatively short period, and longer model simulations with full solar cycle forcing would be needed in order to better evaluate the differences in the response.

\section{Response in shortwave heating rates}

Figure 10 shows the differences in SW heating rates (top) and temperatures (bottom panel) for the month of January in the tropical region, i.e. averaged between $25^{\circ} \mathrm{S}$ and $25^{\circ} \mathrm{N}$. Differences are calculated between January of the year 2004 (during the declining phase of solar cycle 23) and January of 2007, close to solar minimum in December 2008, using the NRLSSI data (solid lines) and the SORCE/SIM and SORCE/SOLSTICE data (dashed lines).

Most of the models listed in the upper part of Tables 2 and 3 performed equilibrium simulations for the years 2004 and 2007. EMAC performed perpetual January runs only, while SOCOL (SOlar Climate Ozone Links) performed transient simulations from 2004 to 2009, with the differences shown here calculated between January of the year 2004 and January of 2007. Therefore most of the displayed differences do not provide one full solar cycle signal. The HadGEM3 (Hadley Centre Global Environmental Model version 3) model simulation used the SORCE/SIM measurements and scaled the data from 2004 to 2007 to obtain a full solar cycle signal (Ineson et al., 2011). GEOSCCM (Goddard Earth Observing System Chemistry-Climate Model) simulations were performed for solar maximum and minimum conditions as well, inferred by a linear fitting approach based on the available SORCE time series (Swartz et al., 2012). The differences presented for HadGEM3 and GEOSCCM are slightly larger than for the other models (and closer to each other), because the simulations correspond to the full solar cycle signal. Please note that SOCOL simulations were performed with the NRLSSI as well as combinations of SORCE/SIM and SORCE/SOLSTICE data (Shapiro et al., 2013b). The simulations performed with three CMIP5 models listed in the lower part of Tables 2 and 3 represent ensemble mean time series experiments with varying solar cycle (e.g. Hardiman et al., 2012). In order to be comparable to the other equilibrium simulations, the differences shown represent the solar signal from a multiple linear regression analysis using the monthly mean TSI or Hartley band irradiance as a solar proxy. Afterwards, the resulting solar signal has been scaled to the 2004-2007 value, i.e. $24 \%$ of the full solar cycle. Note that not all the models were able to provide SW heating rates and/or used interactively calculated ozone. Therefore only a model subset is displayed in the respective figures. In addition to the lines for the single model realisations a multi-model mean (MMM) for three models, namely WACCM (Whole Atmosphere Community Climate Model), GEOSCCM, and HadGEM3 is included in Figs. 10 and 11. As expected, the largest SW heating rate response occurs near the stratopause $(\sim 1 \mathrm{hPa})$ showing the direct impact of solar irradiance variations from 2004-2007. Although the strength of the response differs between the different models, all models show a consistently stronger SW heating (by a factor of up to two to three) using the SORCE data as compared to the NRLSSI data. For both NRLSSI and SORCE simulations, the GEOSCCM shows the strongest response with SW heating rate differences of up to $0.12 \mathrm{Kday}^{-1}$ for NRLSSI compared to $0.42 \mathrm{~K} \mathrm{day}^{-1}$ for SORCE closer to the HadGEM3 response, as both models are depicting full solar 
cycle conditions. In contrast to the other models, HadGEM3, the EMAC-FUB did not take into account solar induced ozone variations. EMAC therefore shows the weakest $\mathrm{SW}$ response in both experiments, followed by the WACCM model. The simulations performed with the CMIP5 models fit nicely to the equilibrium simulations with the same (NRLSSI) forcing. Given the fact that the NRLSSI data provide a lower boundary for SSI variations with the solar cycle, the SW heating rate effects are likely enhanced in nature as compared to recent chemistry-climate model simulations. The SW heating rate differences described above may be attributed to the difference in the radiation codes of the models, as discussed in the previous section, but are also due to the different model set-ups (for example, in simulations performed with or without interactive ozone, the signal will be enhanced or diminished).

\section{Response in temperatures}

Corresponding to the SW heating rate differences, the tropical temperature differences for January are largest near the stratopause; using SORCE, the temperature differences between 2004 and 2007 are three to four times larger than with NRLSSI. Again, the GEOSCCM and the HadGEM models produce the strongest response with about $2 \mathrm{~K}$, and the WACCM and the FUB-EMAC models the weakest response. The response in SOCOL differs slightly from that of the other models by showing the maximum effect around $0.3 \mathrm{hPa}$, i.e. at a higher altitude than the other model simulations. In addition, the temperature response in SOCOL is always weaker using SORCE/SIM or NRLSSI data. When using SORCE/SOLSTICE spectral forcing, the temperature response is approximately half of the response obtained using SORCE/SIM, indicating a large sensitivity of the model to the SSI data sets depicting their differences. Although none of the other models has performed a similar set of simulations, it is expected that this sensitivity is typical for all models shown here. Please note that the negative temperature response in SOCOL in the lower and middle stratosphere is not statistically significant. The temperature responses in the CMIP5 models closely agree with the NRLSSI equilibrium simulations. Comparison of the temperature response to observations over a full $11 \mathrm{yr}$ solar cycle (e.g. Austin et al., 2008; Remsberg, 2008) reveals that the signals derived with the use the NRLSSI data generally show a response peaking around the stratopause, with altitudes of maximum response varying between models according to their model setups as well as the use of solar induced ozone variations. As presented in Chapter 8 of the SPARC-CCMVal Report (2011), where the solar cycle effect on stratospheric temperature from a number of CCM simulations is discussed, the response to the NRLSSI forcing over the full solar cycle is about $0.8 \mathrm{~K}$, similar to observations. The response to the SORCE data forcing is larger than in the observations, but does not scale linearly with larger forcing. It was found to be higher by almost a factor of two in the case of the GEOSCCM simulation which corresponds to a full solar cycle (Swartz et al., 2012).

\section{Response in ozone}

Figure 11 displays the tropical ozone response in percent for month of January for those models that calculate ozone interactively. In the lower stratosphere, ozone changes induced by the forcing with SORCE data are larger and remain positive as compared to those calculated with NRLSSI data. In the middle- to upper-stratosphere ozone differences induced by the NRLSSI data are smaller but still positive, whereas the ozone response using the SORCE data is negative. The height of the change from positive to negative ozone response varies from $5 \mathrm{hPa}$ to $2 \mathrm{hPa}$ between the models. Observations from $8 \mathrm{yr}$ of SABER (Sounding of the Atmosphere using Broadband Emission Radiometry) data indicate a transition altitude of about $1 \mathrm{hPa}$ (Merkel et al., 2011) that is higher than for all models. This change of sign in the ozone response using SORCE data, meaning lower ozone during solar maximum than during solar minimum in the upper stratosphere, is statistically significant in all models. Swartz et al. (2012), who also examined the total ozone response and contributions from heating and photolysis, show good agreement between observations and simulations with the GEOSCCM model. Haigh et al. (2010) showed that the ozone decrease in the upper stratosphere and mesosphere is related to photochemical processes. Decreased $\mathrm{O}_{3}$ at solar maximum is consistent with increased $\mathrm{HO}_{\mathrm{x}}$ and $\mathrm{O}$ and also leads to a selfhealing effect with more UV radiation reaching lower levels, enhancing $\mathrm{O}_{2}$ photolysis and therefore increased $\mathrm{O}_{3}$. Additional observational evidence is needed to confirm the sign reversal in upper atmospheric ozone response.

\section{Dynamical changes and impact on the troposphere}

So far, the discussion focused on the response of stratospheric heating, temperatures and ozone to the forcing with NRLSSI and SORCE data. The direct solar response in the upper stratosphere induces indirect circulation changes throughout the stratosphere (e.g. Kuroda, 2002) and also affects the troposphere and the surface (Haigh, 1999; Kodera, 2002; Matthes et al., 2006). The impact on the troposphere using SORCE data has been reported by Ineson et al. (2011), who provide intriguing but provisional results on Northern Hemispheric winter circulation. Figure 12 displays January multi-model mean signals for three climate models (GEOSCCM, HadGEM3, and WACCM) in zonal mean zonal wind as well as $500 \mathrm{hPa}$ geopotential height using SORCE data as external forcing. A stronger and statistically significant polar night jet during solar maximum years dominates the stratosphere and reaches down to the troposphere, whereas negative wind anomalies that are statistically significant in the lower stratosphere and upper troposphere only 
Table 2. Overview of atmospheric chemistry-climate models and the respective experimental designs using the NRLSSI and/or the SORCE data to study the atmospheric impact.

\begin{tabular}{|c|c|c|c|c|c|c|c|c|}
\hline Model & $\begin{array}{l}\text { Horizontal } \\
\text { resolution }\end{array}$ & Top level & $\begin{array}{l}\text { Number } \\
\text { of } \\
\text { layers }\end{array}$ & $\begin{array}{l}\text { Ozone } \\
\text { interactively } \\
\text { calculated }\end{array}$ & QBO & $\begin{array}{l}\text { Length of } \\
\text { simulation }\end{array}$ & $\begin{array}{l}\text { Equilibrium } \\
\text { simulation }\end{array}$ & Reference \\
\hline \multicolumn{9}{|c|}{ SORCE forcing comparison } \\
\hline $\begin{array}{l}\text { EMAC-CCM } \\
\text { with FUBRad }\end{array}$ & T42 & $0.01 \mathrm{hPa}$ & 39 & no & no & $50 \mathrm{yr}$ & perpetual Jan & Oberländer et al. (2012) \\
\hline $\begin{array}{l}\text { GEOS CCM } \\
\text { (using GEOS5) }\end{array}$ & $2.5^{\circ} \times 2.0^{\circ}$ & $0.01 \mathrm{hPa}$ & 72 & yes & no & $25 \mathrm{yr}$ & yes & Swartz et al. (2012) \\
\hline HadGEM3 & $1.875^{\circ} \times 1.25^{\circ}$ & $85 \mathrm{~km}$ & 85 & no & yes & $20 \times 4$ yr ens & yes & Ineson et al. (2011) \\
\hline IC2-D & $9.5^{\circ}$ lat & $\begin{array}{l}0.26 \mathrm{hPa} \text { for } \\
\text { chemistry }\end{array}$ & $\begin{array}{l}17 \text { for } \\
\text { chemistry }\end{array}$ & yes & no & 670 days & yes & Haigh et al. (2010) \\
\hline SOCOL v 2.0 & T30 & $0.01 \mathrm{hPa}$ & 39 & yes & nudged & 62 months & $\begin{array}{l}\text { transient run } \\
(01.2004- \\
02.2009)\end{array}$ & $\begin{array}{l}\text { Schraner et al. (2008); } \\
\text { Shapiro et al. (2013b) }\end{array}$ \\
\hline WACCM & $2.5^{\circ} \times 1.89^{\circ}$ & $0.000006 \mathrm{hPa}$ & 66 & yes & yes & $25 \mathrm{yr}$ & yes & Merkel et al. (2011) \\
\hline \multicolumn{9}{|c|}{ CMIP5 simulations } \\
\hline HadGEM2 & $1.875^{\circ} \times 1.25^{\circ}$ & $85 \mathrm{Km}$ & 60 & $\begin{array}{l}\text { prescribed solar } \\
\text { induced ozone } \\
\text { variation }\end{array}$ & yes & $\begin{array}{l}3 \text { historical ens. } \\
(1850-2005)\end{array}$ & no & Hardiman et al. (2012) \\
\hline $\begin{array}{l}\text { MPI-ESM-LR } \\
\text { (atmospheric } \\
\text { component: } \\
\text { ECHAM6) }\end{array}$ & T63 & $0.01 \mathrm{hPa}$ & 47 & $\begin{array}{l}\text { prescribed } \\
\text { solar induced } \\
\text { ozone variation }\end{array}$ & no & $\begin{array}{l}3 \text { historical ens. } \\
(1850-2005)\end{array}$ & no & $\begin{array}{l}\text { H. Schmidt } \\
\text { (personal communication, } \\
\text { 2012) }\end{array}$ \\
\hline $\begin{array}{l}\text { WACCM* } \\
\text { (WACCM) }\end{array}$ & $2.5^{\circ} \times 1.89^{\circ}$ & $0.000006 \mathrm{hPa}$ & 66 & yes & nudged & $\begin{array}{l}3 \text { historical ens. } \\
(1960-2005)\end{array}$ & no & Marsh et al. (2013) \\
\hline
\end{tabular}

Table 3. Overview of the spectral range of forcing for the various atmospheric chemistry-climate models employed to study the atmospheric impact.

\begin{tabular}{|c|c|}
\hline Model & Spectral range of forcing \\
\hline \multicolumn{2}{|c|}{ SORCE forcing comparison } \\
\hline \multirow[t]{2}{*}{ EMAC-CCM with FUBRad } & 1) NRLSSI \\
\hline & 2) SIM (SOLSTICE <210 nm) \\
\hline \multirow[t]{3}{*}{ GEOSCCM (using GEOS5) } & 1) NRLSSI \\
\hline & 2) SOLSTICE $<244 \mathrm{~nm}$ and SIM 244 to $1600 \mathrm{~nm}$ \\
\hline & $\begin{array}{l}\text { Solar max and solar min conditions inferred by linear fitting approach based on } \\
\text { the available SORCE time series. }\end{array}$ \\
\hline \multirow[t]{2}{*}{ HadGEM3 } & SIM (only $200-320 \mathrm{~nm})$ \\
\hline & $\begin{array}{l}\text { Extrapolated in time to represent the full solar-cycle amplitude, The estimated } \\
4 \% \text { change is evenly distributed in the UV band. No changes in other bands. }\end{array}$ \\
\hline \multirow[t]{2}{*}{ IC2-D } & 1) NRLSSI \\
\hline & 2) SIM (SOLSTICE <200 nm) \\
\hline \multirow[t]{3}{*}{ SOCOL v 2.0} & 1) NRLSSI \\
\hline & 2) SOCOL SIM (SOLSTICE <210 nm and SIM 210-750 nm) \\
\hline & 3) SOCOL SOLSTICE (SOLSTICE < $290 \mathrm{~nm}$ and SIM 290-750 nm) \\
\hline \multirow[t]{3}{*}{ WACCM } & 1) NRLSSI \\
\hline & 2) SOLSTICE $(116-240 \mathrm{~nm}), \operatorname{SIM}(240-2000 \mathrm{~nm})$ and SRPM $(2000-11000 \mathrm{~nm})$ \\
\hline & CMIP5 simulations \\
\hline HadGEM2 & NRLSSI (CMIP recommended) \\
\hline MPI-ESM-LR (atmospheric component: ECHAM6) & NRLSSI (CMIP recommended) \\
\hline WACCM* CESM1 (WACCM) & NRLSSI (CMIP recommended) \\
\hline
\end{tabular}

dominate equatorwards (Fig. 12, top panel). The zonal wind signals correspond to a positive Arctic oscillation (AO) pattern with a statistically significant stronger polar vortex and a partly statistically significant enhanced geopotential heights in midlatitudes (Fig. 12, bottom panel) in agreement with observations (e.g. Kodera, 2002). The stronger polar vor- tex in the stratosphere leads to a stronger positive phase of AO and North Atlantic Oscillation (NAO), which means a stronger Iceland low and higher pressure of the Azores, and hence an amplified storm track over Europe. This in turn leads to mild conditions over northern Europe and the eastern US and dry conditions in the Mediterranean. However, 

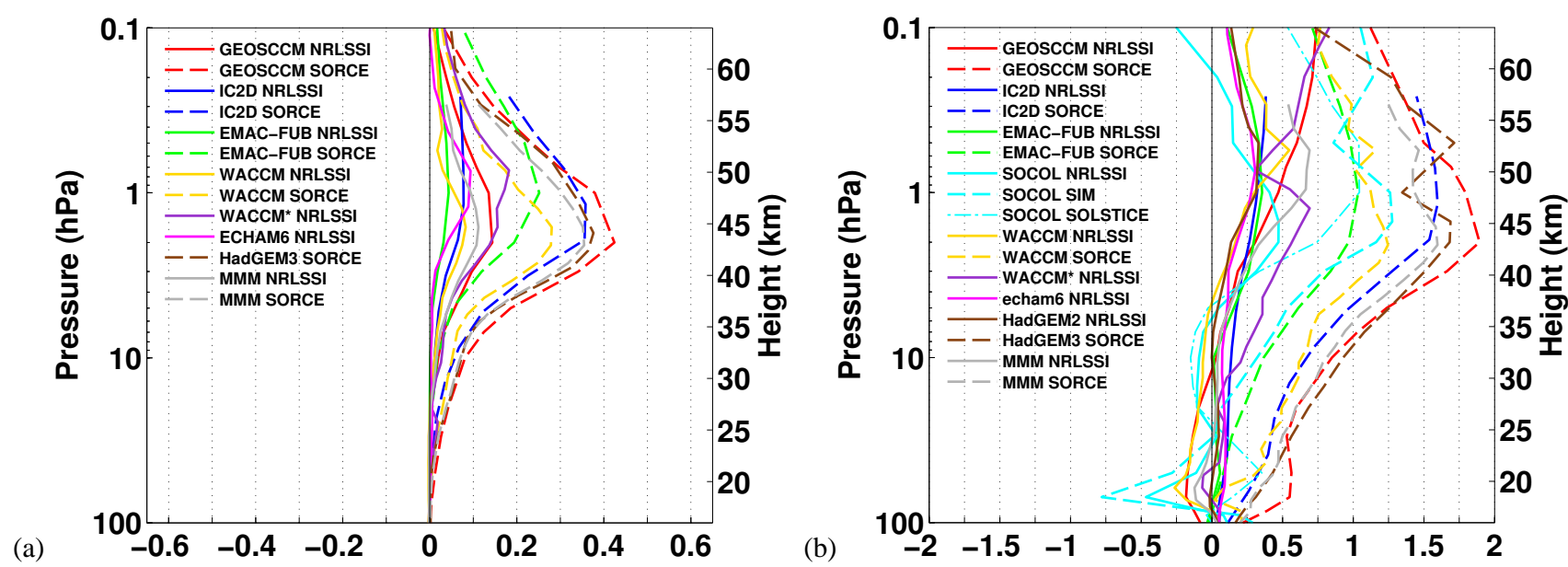

Fig. 10. Differences for January between 2004 and 2007 averaged over the tropics from $25^{\circ} \mathrm{S}$ to $25^{\circ} \mathrm{N}$ for different atmospheric models using the NRLSSI data (solid lines) and the SORCE/SIM and SORCE/SOLSTICE data (dashed lines) for (a) the shortwave heating rate in Kelvin per day $\left(\mathrm{Kday}^{-1}\right)$ and $(\mathbf{b})$ the temperature in Kelvin $(\mathrm{K})$. For details please see text.

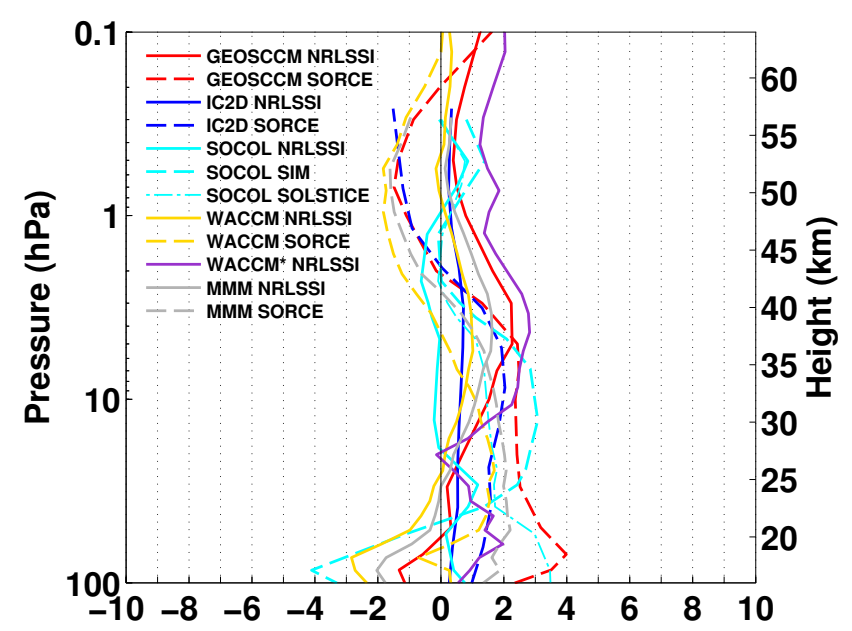

Fig. 11. As Fig. 10 but for the ozone response in percent.

the tropospheric $\mathrm{AO}$ and $\mathrm{NAO}$ already respond to lower UV irradiance variations over the $11 \mathrm{yr}$ cycle, as is shown in Langematz et al. (2013) who obtained a similar AO/NAO response from a transient EMAC-FUB simulation for the period 1960-2005 using NRLSSI data as TOA input. The signal is seen also in Matthes et al. (2006), but in equilibrium simulations with NRLSSI as TOA input. As shown by Ineson et al. (2011), these patterns imply that the solar cycle effect on the $\mathrm{AO} / \mathrm{NAO}$ contributes to a substantial fraction of the typical year-to-year variations and provides therefore a potentially useful source of improved decadal climate predictability for the Northern Hemisphere. Note that the response is regional and is negligible on the global average. However, a caveat is that the $11 \mathrm{yr}$ solar cycle variability cannot be forecasted into the future (on a daily, yearly or decadal timescale).
Even though the solar variability on timescales longer than the $11 \mathrm{yr}$ solar cycle is beyond the scope of this paper, we should note here again that the Sun is the fundamental energy source of the climate system. As such, the low solar activity in the past few years (compared to the previous 6 solar cycles) and its possible implications for future climate evolution has attracted the attention of both scientists and the public (e.g. Lockwood et al., 2010; Schrijver et al., 2011; Jones et al., 2012; Rozanov et al., 2012).

\subsection{Discussion of CCM results}

We described the impact of NRLSSI and SORCE data, which represent the lower and upper boundaries of SSI solar cycle estimated variations, on the atmosphere and climate as depicted in CCM simulations. The NRLSSI reconstructions provide the standard database for simulations of the recent past and future (e.g. SPARC-CCMVal, 2010; Taylor et al., 2012). The atmospheric response with respect to this standard data set is compared to that derived from a different SSI estimate to understand not only the single model responses, but also to point out the importance and robustness of solar cycle signals for climate simulations. In particular, it is worth mentioning that an enhanced spectral resolution in the radiation codes leads to enhanced sensitivity in the response. We also described the important role of solar induced ozone changes for the amplification of solar effects on atmospheric composition, circulation and climate.

Model simulations using the SORCE measurements as compared to the NRLSSI model show larger (by a factor of two) SW heating and temperature signals. The lower irradiance in the visible range during higher solar activity than at minimum activity in the SORCE measurements does not affect the increase in total radiative heating. Recent atmospheric model simulations with enhanced spectral resolution, 

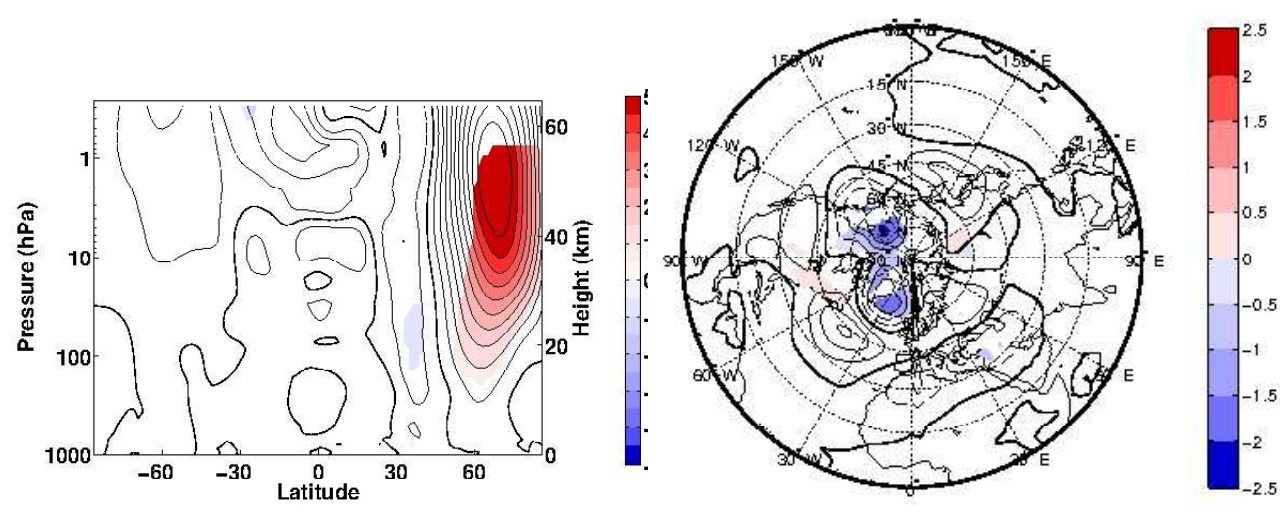

Fig. 12. Multi-model mean (MMM) of the HadGEM3, the GEOS CCM, and the WACCM model for (a) the zonal mean zonal wind differences between 2004 and 2007 in January using the SORCE forcing, and (b) the corresponding geopotential height differences at $500 \mathrm{hPa}$. Coloured areas provide $95 \%$ statistically significant signals.

however, point to the importance of the Chappuis bands in lower stratospheric heating. The solar ozone signal derived when the NRLSSI data are used is positive throughout the stratosphere and mesosphere, whereas the sign is reversed in the upper stratosphere when the SORCE data are used. Observations indicate a reversal of sign in the solar cycle ozone response in the upper atmosphere, however, the transition altitude for the sign change is higher than models suggest. This result needs to be confirmed by other satellite ozone measurements as observational evidence of the solar ozone signal is still limited. Finally, the tropospheric and surface solar cycle response has been presented in an ensemble mean and it has been highlighted that while these solar induced changes are of minor importance for globally averaged temperatures there are larger regional responses.

\section{Conclusions}

This paper presents an overview of our present knowledge of the impact of solar radiative forcing on the Earth's atmosphere. It covers the observations and the modelling of the solar radiative input as well as the modelling of the Earth's atmospheric response. The focus is on satellite-era (i.e. post-1970) data for which direct solar irradiance observations are routinely available. Special attention is given to the role of the UV spectral region, whose small contribution to TSI is compensated by a high relative variability with a potentially amplified influence on climate through radiative heating and ozone photochemistry. There is, today, clear evidence for the impact of solar variability on climate but both its magnitude and its confidence level are still subject to considerable debate. One major challenge lies in the extraction of the weak solar signal from the highly variable atmospheric state. Recent progress has been made along two directions. The first one is the assessment of the magnitude of secular trends in solar radiative forcing through the reconstruction of solar activity on centennial and millennial timescales from indirect indices such as cosmogenic isotopes, (e.g. Solanki et al., 2004; Bard and Frank, 2006; Beer et al., 2006; Usoskin, 2008; Schmidt et al., 2012). The second one, which we focus on, deals with shorter timescales, and is about recent solar variability and its impact on the lower and middle atmosphere. The global physical mechanisms that cause the solar irradiance to change in time and eventually impact climate have been well documented, (e.g. Haigh et al., 2005; Haigh, 2007; Gray et al., 2010; Lean and Woods, 2010, and references therein). More than three decades of SSI observations are now available, but they are highly fragmented and agree poorly because of the difficulties in making radiometrically calibrated and stable measurements from space. Merging these different observations into one single and homogeneous record is a major and ongoing effort. As a consequence, several models have been developed to reproduce the SSI and its variability. The most successful ones are semi-empirical models such as SATIRE, SRPM, and COSI, which describe the SSI in terms of contributions coming from different solar surface magnetic features such as sunspots and faculae. Most models nowadays reproduce SSI measurements on short-term timescales fairly well. However, uncertainties in SSI changes still remain on long-term timescales and in the $220-400 \mathrm{~nm}$ band, which is of particular interest because of its impact on stratospheric ozone. These modelled or observed variations in the SSI are today used as inputs to CCM simulations that are capable of properly reproducing most aspects of stratospheric heating and point to the existence of a significant impact of solar variability on climate. However, major uncertainties remain in their detailed description, in which nonlinear couplings and regional effects can play an important role.

The main topics discussed in this paper and main conclusions of our study are:

- The spectral and temporal coverage of the SSI measurements gradually improved over time, and with the full operation of the SORCE mission in April 2004, daily 
observations of the full UV, visible and NIR spectrum became available. Unfortunately, this situation is likely to end in 2013, when SORCE is anticipated to succumb to battery failure. The lack of SSI observations has led to intensive application of semi-empirical models. There remains a considerable issue in assimilating SSI observations in such models and in reconstructing the SSI prior to the space age. Reconstructions going back to the early 20th century can be derived from semi-empirical models based on historical ground-based solar observations (e.g. Ermolli et al., 2009).

- Recent SSI measurements by SORCE suggest a larger (factor 2-6) variability in the UV (200-400 nm) during solar cycle 23 , which is hard to reconcile with earlier SSI and TSI observations (e.g. by UARS/SOLSTICE and UARS/SUSIM) and with SSI models. However, new estimates of possible calibration corrections suggest that the UV irradiance variation derived from SORCE measurements might be, by almost a factor of two, weaker than reported earlier (Woods, 2012).

- TSI alone does not adequately describe the solar forcing on the atmosphere and therefore SSI variations have to be taken into account in climate models. For many years, the canonical value of the average TSI was $1365.4 \pm 1.3 \mathrm{~W} \mathrm{~m}^{-2}$, whereas now the most accurate, and generally accepted, value is $1361 \pm 0.5 \mathrm{Wm}^{-2}$ (Kopp and Lean, 2011; Schmutz et al., 2012).

- There has been steady progress in modelling SSI variations, and a number of models are now available that can be used as input for climate studies. However, the main uncertainty in the models concerns the wavelength range $220-400 \mathrm{~nm}$, where the magnitude of the variations differs by as much as a factor of three between models. This range is of particular interest for climate studies. Of the five SSI models discussed, specifically NRLSSI, SATIRE-S, COSI, SRPM, and OAR, only one (SRPM) shows a behaviour of the UV and visible irradiance qualitatively resembling that of the recent SORCE/SIM measurements. However, it should be noted that the integral of the SSI computed with SRPM over the entire spectral range (i.e. the TSI) does not reproduce the measured cyclical TSI changes. None of the other four models, which are in closer agreement with each other, reproduces the peculiar behaviour of the UV and visible irradiance observed by SORCE/SIM.

- While there has been major recent progress in better reproducing the SSI changes on short-term timescales, there remains now an important issue in the derivation of realistic confidence intervals, both for the observations and for the model results. Further common metrics should be used for comparing them.
- Within the range of recent SSI values from observations and semi-empirical models, the NRLSSI model and SORCE observations represent, respectively, the lower and upper limits in the magnitude of the solar cycle variation in the UV.

- Results obtained with CCMs show that the observed increase in UV irradiance at solar activity maximum compared to minimum leads to an increase in atmospheric heating rates and, correspondingly, an increase in stratospheric temperatures. This direct atmospheric response is larger for larger UV forcing, i.e. for SORCE as compared to NRLSSI, and is sensitive to resolution in the radiation code. Currently there is insufficient observational evidence to support recent SSI measurements by SORCE on the basis of comparisons between climate model simulations and atmospheric ozone or temperature observations in the stratosphere. The larger UV forcing also leads to a larger surface response. The surface effect is regional and has little influence on globally averaged temperatures.

- Accurate representation of the spectral nature of the incoming radiation, especially at wavelengths below $320 \mathrm{~nm}$, and therefore ozone photochemical variations in the model simulations, is important since these changes amplify the atmospheric solar signal. A more accurate representation of the Chappuis absorption band in a radiation scheme revealed enhanced sensitivity in the heating rate response, which again highlighted the importance of a well resolved radiation code.

A unique aspect of this study is the description of the solar terrestrial connection by an interdisciplinary team of solar and atmospheric physicists. Progress on this hotly debated issue has often been hampered by the fact that limitations on observations or on models are not always properly known outside of a given scientific community. For the first time, a comprehensive comparison and discussion of all relevant SSI measurements and models available for climate studies is presented, as well as a first investigation of their impacts on Earth's climate within a number of different CCMs. These results highlight the importance of taking into account in future climate studies SSI variations and their effects on the Earth's atmosphere.

Major efforts, however, are still needed in each of the three research areas covered in this study. Resolving differences in existing long-term time series of solar measurements is a major challenge; forthcoming initiatives for merging these measurements into single homogenous databases will be of invaluable help (see, e.g. http://projects.pmodwrc.ch/solid/). Overcoming inaccuracies of current SSI models is another important challenge that will benefit from the renewed interest in the solar radiative output, following the recent solar minimum. Finally, the realistic evaluation by climate models of the solar impact on the Earth's climate is an important 
issue. For example, we focussed on the effect of the solar forcing without quantifying the impacts on amplification and feedback mechanisms. This should be done in a coordinated set of CCM experiments where the treatment of SSI inputs to the models are completely specified and results are robustly comparable with each other. Then, it will be also possible to investigate the effects of the top-down feedback and for CCMs with an interactive ocean also the bottom-up feedback mechanism.

Acknowledgements. This work is the outcome of a workshop funded by the COST Action ES1005 TOSCA (http://www. tosca-cost.eu). We thank J. Lean, J. Fontenla, and G. Thuillier for helpful comments and kind collaboration. The authors are grateful to W. Ball, S. Criscuoli, S. Oberländer and M. Kunze for providing updated results of their work, to F. Hansen for preparing Figs. 10 to 12 , and to A. Kubin, G. Chiodo, S. Misios, and A. Maycock for their active participation in the workshop of the COST Action ES1005.

We make the use of SORCE/SIM v17, SORCE/SOLSTICE v12, TIMED/SEE v10, UARS/SOLSTICE v18, UARS/SUSIM v22, SBUV, and SME data from the LISIRD at LASP University of Colorado and members of the instrument teams. Our special thanks are to all instrument teams that made all the observations that were used in this study, as well as to the modelling groups that provided published and partly also unpublished data for this study, in particular to J. Haigh, S. Ineson, D. Marsh, A. Merkel, H. Schmidt, A. V. Shapiro, and W. Swartz. This work is part of the WCRP/SPARC SOLARIS initiative.

E. Rozanov and A. Shapiro are supported by the Swiss National Science Foundation under grant CRSI122-130642 (FUPSOL). S. K. Solanki acknowledges support by the Korean Ministry of Education, Science and Technology (WCU grant No. R31-10016). The work of K. Matthes is supported within the Helmholtz-University Young Investigators Group NATHAN, funded by the Helmholtz Association through the President's Initiative and Networking Fund and the Helmholtz-Zentrum für Ozeanforschung Kiel (GEOMAR). The research leading to some results presented here has received funding also from the EC FP7/2007-2013 Program (project 284461, http://www.eheroes.eu).

We thank the anonymous reviewers, the editor W. Ward, and all participants in the discussion forum for their comments that helped to improve this paper.

Edited by: W. Ward

\section{References}

Abbot, C. G., Fowle, F. E., and Aldrich, L. B.: Chapter VI., Annals of the Astrophysical Observatory of the Smithsonian Institution, 4, 177-215, 1923.

Afram, N., Unruh, Y. C., Solanki, S. K., Schüssler, M., Lagg, A., and Vögler, A.: Intensity contrast from MHD simulations and HINODE observations, Astron. Astrophys., 526, A120, doi:10.1051/0004-6361/201015582, 2011.
Amblard, P.-O., Moussaoui, S., Dudok de Wit, T., Aboudarham, J., Kretzschmar, M., Lilensten, J., and Auchère, F.: The EUV Sun as the superposition of elementary Suns, Astron. Astrophys., 487, L13-L16, doi:10.1051/0004-6361:200809588, 2008.

Austin, J., Tourpali, K., Rozanov, E., Akiyoshi, H., Bekki, S., Bodeker, G., Brühl, C., Butchart, N., Chipperfield, M., Deushi, M., Fomichev, V. I., Giorgetta, M. A., Gray, L., Kodera, K., Lott, F., Manzini, E., Marsh, D., Matthes, K., Nagashima, T., Shibata, K., Stolarski, R. S., Struthers, H., and Tian, W.: Coupled chemistry climate model simulations of the solar cycle in ozone and temperature, J. Geophys. Res.-Atmos., 113, D11306, doi:10.1029/2007JD009391, 2008.

Avrett, E. H. and Loeser, R.: Models of the Solar Chromosphere and Transition Region from SUMER and HRTS Observations: Formation of the Extreme-Ultraviolet Spectrum of Hydrogen, Carbon, and Oxygen, Astrophys. J. Suppl. Ser., 175, 229-276, doi:10.1086/523671, 2008.

Baldwin, M., Dameris, M., Austin, J., Bekki, S., Bregman, B., Butchart, N., Cordero, E., Gillett, N., Graf, H., Granier, C., Kinnison, D., Lal, S., Peter, T., Randel, W., Scinocca, J., Shindell, D., Struthers, H., Takahashi, M., and Thompson, D.: Climate-ozone connections, in: Scientific Assesment of Ozone Depletion: 2006, Global Ozone Research and Monitoring Project Report No. 50, World Meteorological Organization, 2007.

Ball, W. T., Unruh, Y. C., Krivova, N. A., Solanki, S., and Harder, J. W.: Solar irradiance variability: a six-year comparison between SORCE observations and the SATIRE model, Astron. Astrophys., 530, A71, doi:10.1051/0004-6361/201016189, 2011.

Ball, W. T., Unruh, Y. C., Krivova, N. A., Solanki, S., Wenzler, T., Mortlock, D. J., and Jaffe, A. H.: Reconstruction of total solar irradiance 1974-2009, Astron. Astrophys., 541, A27, doi:10.1051/0004-6361/201118702, 2012.

Balmaceda, L. A., Solanki, S. K., Krivova, N. A., and Foster, S.: A homogeneous database of sunspot areas covering more than 130 years, J. Geophys. Res. (Space Physics), 114, A07104, doi:10.1029/2009JA014299, 2009.

Bard, E. and Frank, M.: Climate change and solar variability: What's new under the Sun?, Earth and Planetary Science Letters, 248, 1-14, doi:10.1016/j.epsl.2006.06.016, 2006.

Beer, J., Vonmoos, M., and Muscheler, R.: Solar Variability Over the Past Several Millennia, Space Sci. Rev., 125, 67-79, doi:10.1007/s11214-006-9047-4, 2006.

Bolduc, C., Charbonneau, P., Dumoulin, V., Bourqui, M. S., and Crouch, A. D.: A Fast Model for the Reconstruction of Spectral Solar Irradiance in the Near- and Mid-Ultraviolet, Solar Phys., 279, 383-409, doi:10.1007/s11207-012-0019-4, 2012.

Bovensmann, H., Burrows, J. P., Buchwitz, M., Frerick, J., Noël, S., Rozanov, V. V., Chance, K. V., and Goede, A. P. H.: SCIAMACHY: Mission Objectives and Measurement Modes, J. Atmos. Sci., 56, 127-150, doi:10.1175/15200469(1999)056<0127:SMOAMM>2.0.CO;2, 1999.

Bovensmann, H., Aben, I., van Roozendael, M., Kühl, S., Gottwald, M., von Savigny, C., Buchwitz, M., Richter, A., Frankenberg, C., Stammes, P., de Graaf, M., Wittrock, F., Sinnhuber, B. M., Schönhardt, A., Beirle, S., Gloudemans, A., Schrijver, H., Bracher, A., Rozanov, A. V., Weber, M., and Burrows, J. P.: SCIAMACHY's view of the changing earth's environment, Chap. 10 in: SCIAMACHY - Exploring the Changing Earth's Atmopshere, Springer, Dordrecht, 2011. 
Brasseur, G. P. and Solomon, S.: Aeronomy of the Middle Atmosphere: Chemistry and Physics of the Stratosphere and Mesosphere, in: Aeronomy of the Middle Atmosphere: Chemistry and Physics of the Stratosphere and Mesosphere, by G.P. Brasseur and S. Solomon. 2005 XII, 644 pp. 3rd rev. and enlarged ed. 14020-3284-6, Berlin: Springer, 2005.

Brueckner, G. E., Edlow, K. L., Floyd, IV, L. E., Lean, J. L., and Vanhoosier, M. E.: The solar ultraviolet spectral irradiance monitor (SUSIM) experiment on board the Upper Atmosphere Research Satellite (UARS), J. Geophys. Res., 98, 10695, doi:10.1029/93JD00410, 1993.

Burrows, J. P., Weber, M., Buchwitz, M., Rozanov, V., LadstätterWeißenmayer, A., Richter, A., Debeek, R., Hoogen, R., Bramstedt, K., Eichmann, K.-U., Eisinger, M., and Perner, D.: The Global Ozone Monitoring Experiment (GOME): Mission Concept and First Scientific Results., J. Atmos. Sci., 56, 151-175, doi:10.1175/1520-0469(1999)056<0151:TGOMEG > 2.0.CO;2, 1999.

Butler, J. J., Johnson, B. C., Price, K. P., Shirley, E. L., and Barnes, R. A.: Sources of Differences in On-Orbital Total Solar Irradiance Measurements and Description of a Proposed Laboratory Intercomparison, Journal of Research of the National Institute of Standards and Technology, 113, 187-203, doi:10.6028/jres.113.014, 2008.

Cahalan, R. F., Wen, G., Harder, J. W., and Pilewskie, P.: Temperature responses to spectral solar variability on decadal time scales, Geophys. Res. Lett., 37, L07705, doi:10.1029/2009GL041898, 2010.

Cahalan, R., Pilewskie, P., and Woods, T.: Free flyer Total and Spectral Solar Irradiance Sensor (TSIS) and climate services missions, in: EGU General Assembly Conference Abstracts, edited by: Abbasi, A. and Giesen, N., vol. 14 of EGU General Assembly Conference Abstracts, p. 1886, 2012.

Calisesi, Y., Bonnet, R.-M., Gray, L., Langen, J., and Lockwood, M.: Solar Variability and Planetary Climates, vol. 23 of Space Sciences Series of ISSI, Springer Verlag, Berlin, doi:10.1007/978-0-387-48341-2, 2007.

Carlsson, M. and Stein, R. F.: Formation of Solar Calcium H and K Bright Grains, Astrophys. J., 481, 500, doi:10.1086/304043, 1997.

Cebula, R. P., DeLand, M. T., and Hilsenrath, E.: NOAA 11 solar backscattered ultraviolet, model 2 (SBUV/2) instrument solar spectral irradiance measurements in 1989-1994 1. Observations and long-term calibration, J. Geophys. Res., 103, 16235-16250, doi:10.1029/98JD01205, 1998.

Chapman, G. A., Cookson, A. M., and Dobias, J. J.: Variations in total solar irradiance during solar cycle 22, J. Geophys. Res., 101, 13541-13548, doi:10.1029/96JA00683, 1996.

Chapman, G. A., Cookson, A. M., and Preminger, D. G.: Comparison of TSI from SORCE TIM with SFO Ground-Based Photometry, Solar Phys., 276, 35-41, doi:10.1007/s11207-011-9867-6, 2012.

Coulter, R. L. and Kuhn, J. R.: RISE/PSPT as an Experiment to Study Active Region Irradiance and Luminosity Evolution, in: Solar Active Region Evolution: Comparing Models with Observations, edited by Balasubramaniam, K. S. and Simon, G. W., vol. 68 of Astronomical Society of the Pacific Conference Series, p. 37, 1994.
Crouch, A. D., Charbonneau, P., Beaubien, G., and Paquin-Ricard, D.: A Model for the Total Solar Irradiance Based on Active Region Decay, Astrophys. J., 677, 723-741, doi:10.1086/527433, 2008.

DeLand, M. T. and Cebula, R. P.: Composite MG II solar activity index for solar cycles 21 and 22, J. Geophys. Res., 98, 12809, doi:10.1029/93JD00421, 1993.

DeLand, M. T. and Cebula, R. P.: Creation of a composite solar ultraviolet irradiance data set, J. Geophys. Res. (Space Physics), 113, 11103, doi:10.1029/2008JA013401, 2008.

DeLand, M. T. and Cebula, R. P.: Solar UV variations during the decline of Cycle 23, J. Atmos. Sol.-Terr. Phys., 77, 225-234, doi:10.1016/j.jastp.2012.01.007, 2012.

DeLand, M. T., Floyd, L. E., Rottman, G. J., and Pap, J. M.: Status of UARS solar UV irradiance data, Adv. Space Res., 34, 243250, doi:10.1016/j.asr.2003.03.043, 2004.

Domingo, V., Ermolli, I., Fox, P., Fröhlich, C., Haberreiter, M., Krivova, N., Kopp, G., Schmutz, W., Solanki, S. K., Spruit, H. C., Unruh, Y., and Vögler, A.: Solar Surface Magnetism and Irradiance on Time Scales from Days to the 11-Year Cycle, Space Sci. Rev., 145, 337-380, doi:10.1007/s11214-009-9562-1, 2009.

Donnelly, R. F., Heath, D. F., and Lean, J. L.: Active-region evolution and solar rotation variations in solar UV irradiance, total solar irradiance, and soft X rays, J. Geophys. Res., 87, 1031810324, 1982.

Dudok de Wit, T.: A method for filling gaps in solar irradiance and solar proxy data, Astron. Astrophys., 533, A29, doi:10.1051/0004-6361/201117024, 2011.

Dudok de Wit, T., Kretzschmar, M., Lilensten, J., and Woods, T.: Finding the best proxies for the solar UV irradiance, Geophys. Res. Lett., 36, L10107, doi:10.1029/2009GL037825, 2009.

Eddy, J. A., Gilliland, R. L., and Hoyt, D. V.: Changes in the solar constant and climatic effects, Nature, 300, 689-693, doi:10.1038/300689a0, 1982.

Egorova, T., Rozanov, E., Manzini, E., Haberreiter, M., Schmutz, W., Zubov, V., and Peter, T.: Chemical and dynamical response to the 11-year variability of the solar irradiance simulated with a chemistry-climate model, Geophys. Res. Lett., 31, L06119, doi:10.1029/2003GL019294, 2004.

Ermolli, I., Fofi, M., Bernacchia, C., Berrilli, F., Caccin, B., Egidi, A., and Florio, A.: The prototype RISE-PSPT instrument operating in Rome, Solar Phys., 177, 1-10, 1998.

Ermolli, I., Berrilli, F., and Florio, A.: A measure of the network radiative properties over the solar activity cycle, Astron. Astrophys., 412, 857-864, doi:10.1051/0004-6361:20031479, 2003.

Ermolli, I., Criscuoli, S., Centrone, M., Giorgi, F., and Penza, V.: Photometric properties of facular features over the activity cycle, Astron. Astrophys., 465, 305-314, doi:10.1051/00046361:20065995, 2007.

Ermolli, I., Solanki, S. K., Tlatov, A. G., Krivova, N. A., Ulrich, R. K., and Singh, J.: Comparison Among Ca II K Spectroheliogram Time Series with an Application to Solar Activity Studies, Astrophys. J., 698, 1000-1009, doi:10.1088/0004637X/698/2/1000, 2009.

Ermolli, I., Criscuoli, S., Uitenbroek, H., Giorgi, F., Rast, M. P., and Solanki, S. K.: Radiative emission of solar features in the Ca II $\mathrm{K}$ line: comparison of measurements and models, Astron. Astrophys., 523, A55, doi:10.1051/0004-6361/201014762, 2010. 
Ermolli, I., Criscuoli, S., and Giorgi, F.: Recent results from optical synoptic observations of the solar atmosphere with groundbased instruments, Contributions of the Astronomical Observatory Skalnate Pleso, 41, 73-84, 2011.

Fehlmann, A., Kopp, G., Schmutz, W., Winkler, R., Finsterle, W., and Fox, N.: Fourth World Radiometric Reference to SI radiometric scale comparison and implications for on-orbit measurements of the total solar irradiance, Metrologia, 49, 34, doi:10.1088/0026-1394/49/2/S34, 2012.

Fligge, M. and Solanki, S. K.: The solar spectral irradiance since 1700, Geophys. Res. Lett., 27, 2157-2160, doi:10.1029/2000GL000067, 2000.

Fligge, M., Solanki, S. K., Unruh, Y. C., Fröhlich, C., and Wehrli, C.: A model of solar total and spectral irradiance variations, Astron. Astrophys., 335, 709-718, 1998.

Fligge, M., Solanki, S. K., and Unruh, Y. C.: Modelling irradiance variations from the surface distribution of the solar magnetic field, Astron. Astrophys., 353, 380-388, 2000.

Floyd, L., Rottman, G., DeLand, M., and Pap, J.: 11 years of solar UV irradiance measurements from UARS, in: Solar Variability as an Input to the Earth's Environment, edited by Wilson, A., vol. 535 of ESA Special Publication, 195-203, 2003.

Floyd, L. E., Prinz, D. K., Crane, P. C., and Herring, L. C.: Solar UV irradiance variation during cycles 22 and 23, Adv. Space Res., 29, 1957-1962, doi:10.1016/S0273-1177(02)00242-9, 2002.

Fontenla, J. and Harder, G.: Physical modeling of spectral irradiance variations, Mem. Soc. Astron. It., 76, 826-833, 2005.

Fontenla, J., White, O. R., Fox, P. A., Avrett, E. H., and Kurucz, R. L.: Calculation of Solar Irradiances. I. Synthesis of the Solar Spectrum, Astrophys. J., 518, 480-499, doi:10.1086/307258, 1999.

Fontenla, J. M., Harder, J., Rottman, G., Woods, T. N., Lawrence, G. M., and Davis, S.: The Signature of Solar Activity in the Infrared Spectral Irradiance, Astrophys. J., 605, L85-L88, doi:10.1086/386335, 2004.

Fontenla, J. M., Avrett, E., Thuillier, G., and Harder, J.: Semiempirical Models of the Solar Atmosphere. I. The quiet- and active sun photosphere at moderate resolution, Astrophys. J., 639, 441-458, doi:10.1086/499345, 2006.

Fontenla, J. M., Curdt, W., Haberreiter, M., Harder, J., and Tian, H.: Semiempirical Models of the Solar Atmosphere. III. Set of NonLTE Models for Far-Ultraviolet/Extreme-Ultraviolet Irradiance Computation, Astrophys. J., 707, 482-502, doi:10.1088/0004637X/707/1/482, 2009.

Fontenla, J. M., Harder, J., Livingston, W., Snow, M., and Woods, T.: High-resolution solar spectral irradiance from extreme ultraviolet to far infrared, J. Geophys. Res., 116, D20108, doi:10.1029/2011JD016032, 2011.

Forster, P. M., Fomichev, V. I., Rozanov, E., Cagnazzo, C., Jonsson, A. I., Langematz, U., Fomin, B., Iacono, M. J., Mayer, B., Mlawer, E., Myhre, G., Portmann, R. W., Akiyoshi, H., Falaleeva, V., Gillett, N., Karpechko, A., Li, J., Lemennais, P., Morgenstern, O., Oberländer, S., Sigmond, M., and Shibata, K.: Evaluation of radiation scheme performance within chemistry climate models, J. Geophys. Res.-Atmos., 116, D10302, doi:10.1029/2010JD015361, 2011.

Foukal, P. and Lean, J.: The influence of faculae on total solar irradiance and luminosity, Astrophys. J., 302, 826-835, doi:10.1086/164043, 1986.
Foukal, P. and Lean, J.: An empirical model of total solar irradiance variation between 1874 and 1988, Science, 247, 556-558, doi:10.1126/science.247.4942.556, 1990.

Foukal, P. and Vernazza, J.: The effect of magnetic fields on solar luminosity, Astrophys. J., 234, 707-715, doi:10.1086/157547, 1979.

Foukal, P., Little, R., Graves, J., Rabin, D., and Lynch, D.: Infrared imaging of faculae at the deepest photospheric layers, Solar Phys., 353, 712-715, doi:10.1086/168660, 1990.

Fröhlich, C.: Solar Irradiance Variability Since 1978. Revision of the PMOD Composite during Solar Cycle 21, Space Sci. Rev., 125, 53-65, doi:10.1007/s11214-006-9046-5, 2006.

Fröhlich, C.: Evidence of a long-term trend in total solar irradiance, Astron. Astrophys., 501, L27-L30, doi:10.1051/00046361/200912318, 2009.

Fröhlich, C. and Lean, J.: Total Solar Irradiance Variations: The Construction of a Composite and its Comparison with Models, in: Correlated Phenomena at the Sun, in the Heliosphere and in Geospace, edited by: Wilson, A., vol. 415 of ESA Special Publication, p. 227, 1997.

Fröhlich, C. and Lean, J.: The Sun's total irradiance: Cycles, trends and related climate change uncertainties since 1976, Geophys. Res. Lett., 25, 4377-4380, doi:10.1029/1998GL900157, 1998.

Fröhlich, C. and Lean, J.: Solar radiative output and its variability: evidence and mechanisms, Astron. Astrophys. Rev., 12, 273 320, doi:10.1007/s00159-004-0024-1, 2004.

Fröhlich, C., Andersen, B. N., Appourchaux, T., Berthomieu, G., Crommelynck, D. A., Domingo, V., Fichot, A., Finsterle, W., Gomez, M. F., Gough, D., Jimenez, A., Leifsen, T., Lombaerts, M., Pap, J. M., Provost, J., Cortes, T. R., Romero, J., Roth, H., Sekii, T., Telljohann, U., Toutain, T., and Wehrli, C.: First Results from VIRGO, the Experiment for Helioseismology and Solar Irradiance Monitoring on SOHO, Solar Phys., 170, 1-25, 1997.

Garcia, R. R.: Atmospheric physics: Solar surprise?, Nature, 467, 668-669, doi:10.1038/467668a, 2010.

Gerber, E. P., Baldwin, M. P., Akiyoshi, H., Austin, J., Bekki, S., Braesicke, P., Butchart, N., Chipperfield, M., Dameris, M., Dhomse, S., Frith, S. M., Garcia, R. R., Garny, H., Gettelman, A., Hardiman, S. C., Karpechko, A., Marchand, M., Morgenstern, O., Nielsen, J. E., Pawson, S., Peter, T., Plummer, D. A., Pyle, J. A., Rozanov, E., Scinocca, J. F., Shepherd, T. G., and Smale, D.: Stratosphere-troposphere coupling and annular mode variability in chemistry-climate models, J. Geophys. Res.-Atmos., 115, D00M06, doi:10.1029/2009JD013770, 2010.

Gray, L. J., Beer, J., Geller, M., Haigh, J. D., Lockwood, M., Matthes, K., Cubasch, U., Fleitmann, D., Harrison, G., Hood, L., Luterbacher, J., Meehl, G. A., Shindell, D., van Geel, B., and White, W.: SOLAR INFLUENCES ON CLIMATE, Rev. Geophys., 48, RG4001, doi:10.1029/2009RG000282, 2010.

Grossmann-Doerth, U., Knoelker, M., Schuessler, M., and Solanki, S. K.: The deep layers of solar magnetic elements, Astron. Astrophys., 285, 648-654, 1994.

Haberreiter, M., Krivova, N. A., Schmutz, W., and Wenzler, T.: Reconstruction of solar UV irradiance back to 1974, Adv. Space Res., 35, 365-369, doi:10.1051/0004-6361:200809503, 2005.

Haberreiter, M., Schmutz, W., and Hubeny, I.: NLTE model calculations for the solar atmosphere with an iterative treatment of opacity distribution functions, Astron. Astrophys., 492, 833-840, doi:10.1051/0004-6361:200809503, 2008. 
Haigh, J. D.: The role of stratospheric ozone in modulating the solar radiative forcing of climate, Nature, 370, 544-546, doi:10.1038/370544a0, 1994.

Haigh, J. D.: A GCM study of climate change in response to the 11-year solar cycle, Q. J. Roy. Meteorol. Soc., 125, 871-892, doi:10.1002/qj.49712555506, 1999.

Haigh, J. D.: The Sun and the Earth's Climate, Living Rev. Solar Phys., 4, 64 pp., 2007.

Haigh, J. D., Lockwood, M., and Giampapa, M. S.: The Sun, Solar Analogs and the Climate, Springer Verlag, 2005.

Haigh, J. D., Winning, A. R., Toumi, R., and Harder, J. W.: An influence of solar spectral variations on radiative forcing of climate, Nature, 467, 696-699, doi:10.1038/nature09426, 2010.

Harder, J., Lawrence, G., Fontenla, J., Rottman, G., and Woods, T.: The Spectral Irradiance Monitor: Scientific Requirements, Instrument Design, and Operation Modes, Solar Phys., 230, 141167, doi:10.1007/s11207-005-5007-5, 2005a.

Harder, J. W., Fontenla, J., Lawrence, G., Woods, T., and Rottman, G.: The Spectral Irradiance Monitor: Measurement equations and calibration, Solar Phys., 230, 169-204, doi:10.1007/s11207-0051528-1, 2005b.

Harder, J. W., Fontenla, J. M., Pilewskie, P., Richard, E. C., and Woods, T. N.: Trends in solar spectral irradiance variability in the visible and infrared, Geophys. Res. Lett., 36, L07801, doi:10.1029/2008GL036797, 2009.

Harder, J. W., Thuillier, G., Richard, E. C., Brown, S. W., Lykke, K. R., Snow, M., McClintock, W. E., Fontenla, J. M., Woods, T. N., and Pilewskie, P.: The SORCE SIM solar spectrum: Comparison with recent observations, Solar Phys., 263, 3-24, doi:10.1007/s11207-010-9555-y, 2010.

Hardiman, S., Butchart, N., Hinton, T., Osprey, S., and Gray, L.: The effect of a well resolved stratosphere on surface climate: Differences between CMIP5 simulations with high and low top versions of the Met Office climate model, J. Climate, 25, 70837099, doi:10.1175/JCLI-D-11-00579.1, 2012.

Hickey, J. R., Stowe, L. L., Jacobowitz, H., Pellegrino, P., Maschhoff, R. H., House, F., and Vonder Haar, T. H.: Initial Solar Irradiance Determinations from Nimbus 7 Cavity Radiometer Measurements, Science, 208, 281-283, doi:10.1126/science.208.4441.281, 1980.

Hubeny, I. and Lanz, T.: Non-LTE line-blanketed model atmospheres of hot stars. 1: Hybrid complete linearization/accelerated lambda iteration method, Astrophys. J., 439, 875-904, doi:10.1086/175226, 1995.

Ineson, S., Scaife, A. A., Knight, J. R., Manners, J. C., Dunstone, N. J., Gray, L. J., and Haigh, J. D.: Solar forcing of winter climate variability in the Northern Hemisphere, Nature Geosci., 4, 753757, doi:10.1038/ngeo1282, 2011.

Jain, K. and Hasan, S. S.: Modulation in the solar irradiance due to surface magnetism during cycles 21, 22 and 23, Astron. Astrophys., 425, 301-307, doi:10.1051/0004-6361:20047102, 2004.

Jones, G. S., Lockwood, M., and Stott, P. A.: What influence will future solar activity changes over the 21 st century have on projected global near-surface temperature changes?, J. Geophys. Res.-Atmos., 117, D05103, doi:10.1029/2011JD017013, http://dx.doi.org/10.1029/2011JD017013, 2012.

Keller, C. U., Schüssler, M., Vögler, A., and Zakharov, V.: On the Origin of Solar Faculae, Astrophys. J., 607, L59-L62, doi:10.1086/421553, 2004.
Kobel, P., Solanki, S. K., and Borrero, J. M.: The continuum intensity as a function of magnetic field. I. Active region and quiet Sun magnetic elements, Astron. Astrophys., 531, A112, doi:10.1051/0004-6361/201016255, 2011.

Kodera, K.: Solar cycle modulation of the North Atlantic Oscillation: Implication in the spatial structure of the NAO, Geophys. Res. Lett., 29, 1218, doi:10.1029/2001GL014557, 2002.

Kodera, K. and Kuroda, Y.: Dynamical response to the solar cycle, Journal of Geophysical Research (Atmospheres), 107, 4749, doi:10.1029/2002JD002224, 2002.

Kopp, G. and Lean, J. L.: A new, lower value of total solar irradiance: Evidence and climate significance, Geophys. Res. Lett., 38, L01706, doi:10.1029/2010GL045777, 2011.

Kopp, G., Lawrence, G., and Rottman, G.: The Total Irradiance Monitor (TIM): Science Results, Solar Phys., 230, 129-139, doi:10.1007/s11207-005-7433-9, 2005.

Krivova, N. and Solanki, S.: Models of Solar Total and Spectral Irradiance Variability of Relevance for Climate Studies, in: Climate and Weather of the Sun-Earth System (CAWSES), edited by: Lübken, F.-J., Springer Atmospheric Sciences, 19-38, Springer, the Netherlands, doi:10.1007/978-94-007-4348-9_2, 2013.

Krivova, N. A., Solanki, S. K., Fligge, M., and Unruh, Y. C.: Reconstruction of solar irradiance variations in cycle 23: Is solar surface magnetism the cause?, Astron. Astrophys., 399, L1-L4, doi:10.1051/0004-6361:20030029, 2003.

Krivova, N. A., Solanki, S. K., and Floyd, L.: Reconstruction of solar UV irradiance in cycle 23, Astron. Astrophys., 452, 631639, doi:10.1051/0004-6361:20064809, 2006.

Krivova, N. A., Balmaceda, L., and Solanki, S. K.: Reconstruction of solar total irradiance since 1700 from the surface magnetic flux, Astron. Astrophys., 467, 335-346, doi:10.1051/00046361:20066725, 2007.

Krivova, N. A., Solanki, S. K., Wenzler, T., and Podlipnik, B.: Reconstruction of solar UV irradiance since 1974, J. Geophys. Res.Atmos., 114, D00I04, doi:10.1029/2009JD012375, 2009.

Krivova, N. A., Vieira, L. E. A., and Solanki, S. K.: Reconstruction of solar spectral irradiance since the Maunder minimum, J. Geophys. Res. (Space Physics), 115, A12112, doi:10.1029/2010JA015431, 2010.

Kuroda, Y.: Relationship between the Polar-Night Jet Oscillation and the Annular Mode, Geophys. Res. Lett., 29, 1240, doi:10.1029/2001GL013933, 2002.

Kurucz, R.: ATLAS9 Stellar Atmosphere Programs and $2 \mathrm{~km} / \mathrm{s}$ grid., ATLAS9 Stellar Atmosphere Programs and $2 \mathrm{~km} / \mathrm{s}$ grid. Kurucz CD-ROM No. 13. Cambridge, Mass.: Smithsonian Astrophysical Observatory, 13, 1993.

Kurucz, R. L.: New Opacity Calculations, in: NATO ASIC Proc. 341: Stellar Atmospheres - Beyond Classical Models, edited by: Crivellari, L., Hubeny, I., and Hummer, D. G., p. 441, 1991.

Kurucz, R. L.: ATLAS12, SYNTHE, ATLAS9, WIDTH9, et cetera, Memorie della Societa Astronomica Italiana Supplementi, 8, 14, 2005.

Langematz, U., Kubin, A., Brühl, C., Baumgaertner, A., Cubasch, U., and Spangehl, T.: Solar Effects on Chemistry and Climate Including Ocean Interactions, in: Climate and Weather of the Sun-Earth System (CAWSES), edited by: Lübken, F.-J., Springer Atmospheric Sciences, 541-571, Springer Netherlands, doi:10.1007/978-94-007-4348-9_29, 2013. 
Lawrence, G. M., Rottman, G., Harder, J., and Woods, T.: Solar Total Irradiance Monitor (TIM), Metrologia, 37, 407, doi:10.1088/0026-1394/37/5/13, 2000.

Lawrence, G. M., Kopp, G., Rottman, G., Harder, J., Woods, T., and H Loui: Calibration of the total irradiance monitor, Metrologia, 40, S78-S80, doi:10.1088/0026-1394/40/1/317, 2003.

Lean, J.: The Sun's Variable Radiation and Its Relevance For Earth, Annu. Rev. Astron. Astrophys., 35, 33-67, doi:10.1146/annurev.astro.35.1.33, 1997.

Lean, J. L.: Evolution of the Sun's spectral irradiance since the Maunder Minimum, Geophys. Res. Lett., 27, 2425-2428, doi:10.1029/2000GL000043, 2000.

Lean, J. L. and DeLand, M. T.: How Does the Sun's Spectrum Vary?, J. Climate, 25, 2555-2560, doi:10.1175/JCLI-D-11$00571.1,2012$.

Lean, J. L. and Woods, T. N.: Solar total and spectral irradiance measurements and models, in: Evolving Solar Physics and the Climates of Earth and Space, edited by Schrijver, C. J. and Siscoe, G. L., Cambridge Univeristy Press, Cambridge, 2010.

Lean, J. L., Livingston, W. C., Heath, D. F., Donnelly, R. F., Skumanich, A., and White, O. R.: A three-component model of the variability of the solar ultraviolet flux 145-200 nM, J. Geophys. Res., 87, 10307-10317, doi:10.1029/JA087iA12p10307, 1982.

Lean, J. L., Rottman, G. J., Kyle, H. L., Woods, T. N., Hickey, J. R., and Puga, L. C.: Detection and parameterization of variations in solar mid- and near-ultraviolet radiation (200-400 nm), J. Geophys. Res., 102, 29939-29956, doi:10.1029/97JD02092, 1997.

Lee, III, R. B., Barkstrom, B. R., and Cess, R. D.: Characteristics of the earth radiation budget experiment solar monitors, Appl. Optics, 26, 3090-3096, doi:10.1364/AO.26.003090, 1987.

Lee, III, R. B., Gibson, M. A., Wilson, R. S., and Thomas, S.: Longterm total solar irradiance variability during sunspot cycle 22, J. Geophys. Res., 100, 1667-1675, doi:10.1029/94JA02897, 1995.

Lockwood, M.: Was UV spectral solar irradiance lower during the recent low sunspot minimum?, J. Geophys. Res.-Atmos., 116, D16103, doi:10.1029/2010JD014746, 2011.

Lockwood, M.: Solar Influence on Global and Regional Climates, Surveys in Geophysics, p. 27, doi:10.1007/s10712-012-9181-3, 2012.

Lockwood, M., Harrison, R. G., Woollings, T., and Solanki, S. K.: Are cold winters in Europe associated with low solar activity?, Environ. Res. Lett., 5, 024001, doi:10.1088/17489326/5/2/024001, 2010.

Loukitcheva, M., Solanki, S. K., Carlsson, M., and Stein, R. F.: Millimeter observations and chromospheric dynamics, Astron. Astrophys., 419, 747-756, doi:10.1051/0004-6361:20034159, 2004.

Marsh, D. R., Mills, M. J., Kinnison, D. E., Lamarque, J. F., Calvo, N., and Polvani, L.: Climate change from 1850 to 2005 simulated in CESM1 (WACCM), J. Climate, in review, 2013.

Matthes, K.: Atmospheric science: Solar cycle and climate predictions, Nature Geosci., 4, 735-736, doi:10.1038/ngeo1298, 2011.

Matthes, K., Kuroda, Y., Kodera, K., and Langematz, U.: Transfer of the solar signal from the stratosphere to the troposphere: Northern winter, J. Geophys. Res.-Atmos., 111, D06108, doi:10.1029/2005JD006283, 2006.

McClintock, W. E., Rottman, G. J., and Woods, T. N.: SolarStellar Irradiance Comparison Experiment II (Solstice II): Instrument Concept and Design, Solar Phys., 230, 225-258, doi:10.1007/s11207-005-7432-x, 2005.

Meehl, G., Arblaster, J., Matthes, K., Sassi, F., and van Loon, H.: Amplifying the Pacific climate system response to a small 11 year solar cycle forcing, Science, 325, 1114-1118, doi:10.1126/science.1172872, 2009.

Merkel, A. W., Harder, J. W., Marsh, D. R., Smith, A. K., Fontenla, J. M., and Woods, T. N.: The impact of solar spectral irradiance variability on middle atmospheric ozone, Geophys. Res. Lett., 38, L13802, doi:10.1029/2011GL047561, 2011.

Morrill, J. S., Floyd, L., and McMullin, D.: The Solar Ultraviolet Spectrum Estimated Using the Mg ii Index and Ca ii K Disk Activity, Solar Phys., 269, 253-267, doi:10.1007/s11207-011-97087, 2011a.

Morrill, J. S., Floyd, L. E., and McMullin, D. R.: Solar UV Spectral Irradiance Measured by SUSIM During Solar Cycle 22 and 23, AGU Fall Meeting Abstracts, p. A914, 2011b.

Nissen, K. M., Matthes, K., Langematz, U., and Mayer, B.: Towards a better representation of the solar cycle in general circulation models, Atmos. Chem. Phys., 7, 5391-5400, doi:10.5194/acp-75391-2007, 2007.

Oberländer, S., Langematz, U., Matthes, K., Kunze, M., Kubin, A., Harder, J., Krivova, N. A., Solanki, S. K., Pagaran, J., and Weber, M.: The influence of spectral solar irradiance data on stratospheric heating rates during the 11 year solar cycle, Geophys. Res. Lett., 39, L01801, doi:10.1029/2011GL049539, 2012.

Oster, L., Schatten, K. H., and Sofia, S.: Solar irradiance variations due to active regions, Astrophys. J., 256, 768-773, doi:10.1086/159949, 1982.

Pagaran, J., Weber, M., and Burrows, J.: Solar Variability from 240 to $1750 \mathrm{~nm}$ in Terms of Faculae Brightening and Sunspot Darkening from SCIAMACHY, Astrophys. J., 700, 1884-1895, doi:10.1088/0004-637X/700/2/1884, 2009.

Pagaran, J., Harder, J. W., Weber, M., Floyd, L. E., and Burrows, J. P.: Intercomparison of SCIAMACHY and SIM vis-IR irradiance over several solar rotational timescales, Astron. Astrophys., 528, A67, doi:10.1051/0004-6361/201015632, 2011a.

Pagaran, J., Weber, M., DeLand, M. T., Floyd, L. E., and Burrows, J. P.: Solar Spectral Irradiance Variations in 240-1600 nm During the Recent Solar Cycles 21-3, Solar Phys., 272, 159-188, doi:10.1007/s11207-011-9808-4, 2011b.

Pap, J. M., Willson, R. C., Froelich, C., Donnelly, R. F., and Puga, L.: Long-term variations in total solar irradiance, Solar Phys., 152, 13-21, doi:10.1007/BF01473177, 1994.

Pap, J. M., Fox, P., Fröhlich, C., Hudson, H. S., Kuhn, J., McCormack, J., North, G., Sprigg, W., and Wu, S. T. (Eds.): Solar Variability and its Effects on Climate, vol. 141 of Geophysical Monograph Series, American Geophysical Union, Washington DC, 2004.

Penza, V., Caccin, B., Ermolli, I., Centrone, M., and Gomez, M. T.: Modeling solar irradiance variations through PSPT images and semiempirical models, in: Solar Variability as an Input to the Earth's Environment, edited by: Wilson, A., vol. 535 of ESA Special Publication, 299-302, 2003.

Penza, V., Caccin, B., Ermolli, I., and Centrone, M.: Comparison of model calculations and photometric observations of bright "magnetic" regions, Astron. Astrophys., 413, 1115-1123, doi:10.1051/0004-6361:20031397, 2004.

Penza, V., Pietropaolo, E., and Livingston, W.: Modeling the cyclic modulation of photospheric lines, Astron. Astrophys., 454, 349- 
358, doi:10.1051/0004-6361:20053405, 2006.

Preminger, D. G., Walton, S. R., and Chapman, G. A.: Photometric quantities for solar irradiance modeling, J. Geophys. Res. (Space Physics), 107, 1354, doi:10.1029/2001JA009169, 2002.

Remsberg, E. E.: On the response of Halogen Occultation Experiment (HALOE) stratospheric ozone and temperature to the 11year solar cycle forcing, J. Geophys. Res.-Atmos., 113, D22304, doi:10.1029/2008JD010189, 2008.

Röhrbein, D., Cameron, R., and Schüssler, M.: Is there a nonmonotonic relation between photospheric brightness and magnetic field strength in solar plage regions?, Astron. Astrophys., 532, A140, doi:10.1051/0004-6361/201117090, 2011.

Rottman, G.: The SORCE Mission, Solar Phys., 230, 7-25, doi:10.1007/s11207-005-8112-6, 2005.

Rottman, G., Woods, T., Snow, M., and Detoma, G.: The solar cycle variation in ultraviolet irradiance, Adv. Space Res., 27, 19271932, doi:10.1016/S0273-1177(01)00272-1, 2001.

Rottman, G., Floyd, L., and Viereck, R.: Measurement of the Solar Ultraviolet Irradiance, in: Solar Variability and its Effects on Climate. Geophysical Monograph 141, edited by Pap, J. M., Fox, P., Fröhlich, C., Hudson, H. S., Kuhn, J., McCormack, J., North, G., Sprigg, W., and Wu, S. T., vol. 141 of Washington DC American Geophysical Union Geophysical Monograph Series, 111125, 2004.

Rottman, G. J., Woods, T. N., and Sparn, T. P.: Solar-Stellar Irradiance Comparison Experiment 1. I - Instrument design and operation, J. Geophys. Res., 98, 10667, doi:10.1029/93JD00462, 1993.

Rozanov, E., Egorova, T., Fröhlich, C., Haberreiter, M., Peter, T., and Schmutz, W.: Estimation of the ozone and temperature sensitivity to the variation of spectral solar flux, in: From Solar Min to Max: Half a Solar Cycle with SOHO, edited by: Wilson, A., vol. 508 of ESA Special Publication, pp. 181-184, 2002.

Rozanov, E. V., Egorova, T. A., Shapiro, A. I., and Schmutz, W. K.: Modeling of the atmospheric response to a strong decrease of the solar activity, in: IAU Symposium, vol. 286 of IAU Symposium, pp. 215-224, doi:10.1017/S1743921312004863, 2012.

Rutten, R. J.: Radiative Transfer in Stellar Atmospheres, University of Utrecht, Uttecht, the Netherlands, 2003.

Rutten, R. J. and Kostik, R. I.: Empirical NLTE analyses of solar spectral lines. III - Iron lines versus LTE models of the photosphere, Astron. Astrophys., 115, 104-114, 1982.

Schmidt, G. A., Jungclaus, J. H., Ammann, C. M., Bard, E., Braconnot, P., Crowley, T. J., Delaygue, G., Joos, F., Krivova, N. A., Muscheler, R., Otto-Bliesner, B. L., Pongratz, J., Shindell, D. T., Solanki, S. K., Steinhilber, F., and Vieira, L. E. A.: Climate forcing reconstructions for use in PMIP simulations of the Last Millennium (v1.1), Geosci. Model Dev., 5, 185-191, doi:10.5194/gmd-5-185-2012, 2012.

Schmutz, W., Fehlmann, A., Hülsen, G., Meindl, P., Winkler, R., Thuillier, G., Blattner, P., Buisson, F., Egorova, T., Finsterle, W., Fox, N., Gröbner, J., Hochedez, J.-F., Koller, S., Meftah, M., Meisonnier, M., Nyeki, S., Pfiffner, D., Roth, H., Rozanov, E., Spescha, M., Wehrli, C., Werner, L., and Wyss, J. U.: The PREMOS/PICARD instrument calibration, Metrologia, 46, 202, doi:10.1088/0026-1394/46/4/S13, 2009.

Schmutz, W., Fehlmann, A., Finsterle, W., and the PREMOS team: First light of PREMOS/PICARD, Annual report 2011, p. 44, PMOD/WRC, www.pmodwrc.ch, 2012.
Schraner, M., Rozanov, E., Schnadt Poberaj, C., Kenzelmann, P., Fischer, A. M., Zubov, V., Luo, B. P., Hoyle, C. R., Egorova, T., Fueglistaler, S., Brönnimann, S., Schmutz, W., and Peter, T.: Technical Note: Chemistry-climate model SOCOL: version 2.0 with improved transport and chemistry/microphysics schemes, Atmos. Chem. Phys., 8, 5957-5974, doi:10.5194/acp-8-59572008, 2008.

Schrijver, C. J., Livingston, W. C., Woods, T. N., and Mewaldt, R. A.: The minimal solar activity in 2008-2009 and its implications for long-term climate modeling, Geophys. Res. Lett., 38, L06701, doi:10.1029/2011GL046658, 2011.

Shapiro, A., Schmutz, W., Dominique, M., and Shapiro, A.: Eclipses observed by LYRA - a sensitive tool to test the models for the solar irradiance, Solar Phys., in press, 2013a.

Shapiro, A. I., Schmutz, W., Schoell, M., Haberreiter, M., and Rozanov, E.: NLTE solar irradiance modeling with the COSI code, Astron. Astrophys., 517, A48, doi:10.1051/00046361/200913987, 2010.

Shapiro, A. I., Schmutz, W., Rozanov, E., Schoell, M., Haberreiter, M., Shapiro, A. V., and Nyeki, S.: A new approach to the long-term reconstruction of the solar irradiance leads to large historical solar forcing, Astron. Astrophys., 529, A67, doi:10.1051/0004-6361/201016173, 2011.

Shapiro, A. V., Rozanov, E. V., Shapiro, A. I., Egorova, T. A., Harderi, J., Weber, M., Smith, A. K., Schmutz, W., and Peter, T.: The role of the solar irradiance variability in the evolution of the middle atmosphere during 2004-2009, J. Geophys. Res.-Atmos., doi:10.1002/jgrd.50208, in press, 2013b.

Shapiro, A. V., Shapiro, A. I., Dominique, M., Dammasch, I. E., Wehrli, C., Rozanov, E., and Schmutz, W.: Detection of Solar Rotational Variability in the Large Yield RAdiometer (LYRA) 190-222 nm Spectral Band, Solar Phys., p. 121, doi:10.1007/s11207-012-0029-2, 2012.

Shchukina, N. and Trujillo Bueno, J.: The Iron Line Formation Problem in Three-dimensional Hydrodynamic Models of Solar-like Photospheres, Astrophys. J., 550, 970-990, doi:10.1086/319789, 2001.

Snow, M., McClintock, W. E., Rottman, G., and Woods, T. N.: Solar Stellar Irradiance Comparison Experiment II (Solstice II): Examination of the Solar Stellar Comparison Technique, Solar Phys., 230, 295-324, doi:10.1007/s11207-005-8763-3, 2005.

Socas-Navarro, H.: A high-resolution three-dimensional model of the solar photosphere derived from Hinode observations, Astron. Astrophys., 529, A37, doi:10.1051/0004-6361/201015805, 2011.

Solanki, S. K. and Unruh, Y. C.: A model of the wavelength dependence of solar irradiance variations, Astron. Astrophys., 329, 747-753, 1998.

Solanki, S. K., Usoskin, I. G., Kromer, B., Schüssler, M., and Beer, J.: Unusual activity of the Sun during recent decades compared to the previous 11,000 years, Nature, 431, 1084-1087, doi:10.1038/nature02995, 2004.

Solanki, S. K., Krivova, N. A., and Haigh, J. D.: Solar Activity and Climate, Annu. Rev. Astron. Astrophys., 51, http://www.annualreviews.org/doi/abs/10.1146/ annurev-astro-082812-141007, 2013.

Solomon, S. D., Qin, D., Manning, M., Chen, Z., Marquis, M., Averyt, K. B., Tignor, M., and Miller, H.: Climate Change 2007: The Physical Science Basis (Contribution of Working Group I to 
the Fourth Assessment Report of the Intergovernmental Panel on Climate Change), Cambridge Univeristy Press, Cambridge, UK, 2007.

SPARC-CCMVal: SPARC Report on the Evaluation of ChemistryClimate Models, SPARC Report No. 5, WCRP-132, WMO/TDNo. 1526, edited by: Eyring, V., Shepherd, T. G., and Waugh, D. W., www.atmosp.physics.utoronto.ca/SPARC (last access: May 2011), 2010.

Sperfeld, P., Metzdorf, J., Galal Yousef, S., Stock, K. D., and Möller, W.: Improvement and extension of the black-body-based spectral irradiance scale, Metrologia, 35, 267, doi:10.1088/00261394/35/4/9, 1998.

Spruit, H. C.: Pressure equilibrium and energy balance of small photospheric fluxtubes, Solar Phys., 50, 269-295, doi:10.1007/BF00155292, 1976.

Spruit, H. C.: The flow of heat near a starspot, Astron. Astrophys., 108, 356-360, 1982.

Swartz, W. H., Stolarski, R. S., Oman, L. D., Fleming, E. L., and Jackman, C. H.: Middle atmosphere response to different descriptions of the 11-yr solar cycle in spectral irradiance in a chemistry-climate model, Atmos. Chem. Phys., 12, 5937-5948, doi:10.5194/acp-12-5937-2012, 2012.

Taylor, K. E., Stouffer, R. J., and Meehl, G. A.: An Overview of CMIP5 and the Experiment Design, B. Am. Meteorol. Soc., 93, 485-498, doi:10.1175/BAMS-D-11-00094.1, 2012.

Thuillier, G., Herse, M., Simon, P. C., Labs, D., Mandel, H., Gillotay, D., and Foujols, T.: The Visible Solar Spectral Irradiance from 350 to $850 \mathrm{NM}$ as Measured by the SOLSPEC Spectrometer During the Atlas I Mission, Solar Phys., 177, 41-61, 1998.

Thuillier, G., Floyd, L., Woods, T. N., Cebula, R., Hilsenrath, E., Hersé, M., and Labs, D.: Solar Irradiance Reference Spectra, in: Solar Variability and its Effects on Climate. Geophysical Monograph 141, edited by Pap, J. M., Fox, P., Fröhlich, C., Hudson, H. S., Kuhn, J., McCormack, J., North, G., Sprigg, W., and Wu, S. T., vol. 141 of Washington DC American Geophysical Union Geophysical Monograph Series, 171-194, 2004.

Thuillier, G., Foujols, T., Bolsée, D., Gillotay, D., Hersé, M., Peetermans, W., Decuyper, W., Mandel, H., Sperfeld, P., Pape, S., Taubert, D. R., and Hartmann, J.: SOLAR/SOLSPEC: Scientific Objectives, Instrument Performance and Its Absolute Calibration Using a Blackbody as Primary Standard Source, Solar Phys., 257, 185-213, doi:10.1007/s11207-009-9361-6, 2009.

Thuillier, G., Claudel, J., Djafer, D., Haberreiter, M., Mein, N., Melo, S. M. L., Schmutz, W., Shapiro, A., Short, C. I., and Sofia, S.: The Shape of the Solar Limb: Models and Observations, Solar Phys., 268, 125-149, doi:10.1007/s11207-010-9664-7, 2011.

Thuillier, G., DeLand, M., Shapiro, A., Schmutz, W., Bolsée, D., and Melo, S. M. L.: The Solar Spectral Irradiance as a Function of the $\mathrm{Mg}$ ii Index for Atmosphere and Climate Modelling, Solar Phys., 277, 245-266, doi:10.1007/s11207-011-9912-5, 2012.

Topka, K. P., Tarbell, T. D., and Title, A. M.: Properties of the smallest solar magnetic elements. I - Facular contrast near sun center, Astrophys. J., 396, 351-363, doi:10.1086/171721, 1992.

Trenberth, K. E., Fasullo, J. T., and Kiehl, J.: Earth's Global Energy Budget, B. Am. Meteorol. Soc., 90, 311-324, doi:10.1175/2008BAMS2634.1, 2009.

Uitenbroek, H.: Multilevel Radiative Transfer with Partial Frequency Redistribution, Astrophys. J., 557, 389-398, doi:10.1086/321659, 2001.

Uitenbroek, H.: The Effect of Coherent Scattering on Radiative Losses in the Solar Ca II K Line, Astrophys. J., 565, 1312-1322, doi:10.1086/324698, 2002.

Uitenbroek, H. and Criscuoli, S.: Why One-dimensional Models Fail in the Diagnosis of Average Spectra from Inhomogeneous Stellar Atmospheres, Astrophys. J., 736, 69, doi:10.1088/0004637X/736/1/69, 2011.

Unruh, Y. C., Solanki, S. K., and Fligge, M.: The spectral dependence of facular contrast and solar irradiance variations, Astron. Astrophys., 345, 635-642, 1999.

Unruh, Y. C., Krivova, N. A., Solanki, S. K., Harder, J. W., and Kopp, G.: Spectral irradiance variations: comparison between observations and the SATIRE model on solar rotation time scales, Astron. Astrophys., 486, 311-323, doi:10.1051/00046361:20078421, 2008.

Unruh, Y. C., Ball, W. T., and Krivova, N. A.: Solar Irradiance Models and Measurements: A Comparison in the 220$240 \mathrm{~nm}$ wavelength band, Surveys in Geophysics, 33, 475-481, doi:10.1007/s10712-011-9166-7, 2012.

Usoskin, I. G.: A History of Solar Activity over Millennia, Living Reviews in Solar Physics, 5, http://solarphysics.livingreviews. org/Articles/lrsp-2008-3/, 2008.

van Loon, H., Meehl, G. A., and Shea, D.: Coupled air-sea response to solar forcing in the Pacific region during northern winter, J. Geophys. Res., 112, D02108, doi:10.1029/2006JD007378, 2007.

Vernazza, J. E., Avrett, E. H., and Loeser, R.: Structure of the solar chromosphere. III - Models of the EUV brightness components of the quiet-sun, Astrophys. J. Suppl. Ser., 45, 635-725, doi:10.1086/190731, 1981.

Vieira, L. E. A., Solanki, S. K., Krivova, N. A., and Usoskin, I.: Evolution of the solar irradiance during the Holocene, Astron. Astrophys., 531, A6, doi:10.1051/0004-6361/201015843, 2011.

Viereck, R., Puga, L., McMullin, D., Judge, D., Weber, M., and Tobiska, W. K.: The Mg II index: A proxy for solar EUV, Geophys. Res. Lett., 28, 1343-1346, doi:10.1029/2000GL012551, 2001.

Vögler, A.: On the effect of photospheric magnetic fields on solar surface brightness, Results of radiative MHD simulations, Memorie della Società Astronomica Italianasai, 76, 842-849, 2005.

Wang, H., Spirock, T., Goode, P., Lee, C., Zirin, H., and Kosonocky, W.: Contrast of faculae at 1.6 mMicrons, Astrophys. J., 495, 957964, doi:10.1086/305311, 1998.

Weber, M., Burrows, J. P., and Cebula, R. P.: Gome Solar UV/VIS Irradiance Measurements between 1995 and 1997 - First Results on Proxy Solar Activity Studies, Solar Phys., 177, 63-77, 1998.

Weber, M., Pagaran, J., Dikty, S., von Savigny, C., Burrows, J., DeLand, M., Floyd, L., Harder, J., Mlynczak, M., and Schmidt, H.: Investigation of solar irradiance variations and their impact on middle atmospheric ozone, in: Climate and Weather of the Sun-Earth System (CAWSES), edited by: Lubken, F.-J., Springer Atmospheric Sciences, 39-54, Springer, the Netherlands, doi:10.1007/978-94-007-4348-9_3, 2013.

Wehrli, C., Schmutz, W., and Shapiro, A.: Correlation of Spectral Solar Irradiance with solar activity as measured by SPM/VIRGO, Astron. Astrophys., submitted, 2012.

Wenzler, T., Solanki, S. K., Krivova, N. A., and Fluri, D. M.: Comparison between KPVT/SPM and SoHO/MDI magnetograms with an application to solar irradiance reconstructions, Astron. Astrophys., 427, 1031-1043, doi:10.1051/0004-6361:20041313, 
2004.

Wenzler, T., Solanki, S. K., and Krivova, N. A.: Can surface magnetic fields reproduce solar irradiance variations in cycles 22 and 23?, Astron. Astrophys., 432, 1057-1061, doi:10.1051/00046361:20041956, 2005.

Wenzler, T., Solanki, S. K., Krivova, N. A., and Fröhlich, C.: Reconstruction of solar irradiance variations in cycles 21-23 based on surface magnetic fields, Astron. Astrophys., 460, 583-595, doi:10.1051/0004-6361:20065752, 2006.

Wenzler, T., Solanki, S. K., and Krivova, N. A.: Reconstructed and measured total solar irradiance: Is there a secular trend between 1978 and 2003?, Geophys. Res. Lett., 36, L11102, doi:10.1029/2009GL037519, 2009.

Willson, R. C.: Active cavity radiometer type IV, Appl. Optics, 18, 179-188, doi:10.1364/AO.18.000179, 1979.

Willson, R. C.: The ACRIMSAT/ACRIM III experiment: extending the precision, long-term total solar irradiance climate database, Earth Observer, 13, 14-17, 2001.

Willson, R. C., Gulkis, S., Janssen, M., Hudson, H. S., and Chapman, G. A.: Observations of solar irradiance variability, Science, 211, 700-702, doi:10.1126/science.211.4483.700, 1981.

WMO: Global Ozone Research and Monitoring Project Report No. 52, in: Scientific Assessment of Ozone Depletion: 2010, p. 516, World Meteorological Organisation, Geneva, Switzerland, 2011.
Woods, T.: Solar Irradiance Variability: Comparisons of Observations over Solar Cycles 21-24, in: EGU General Assembly Conference Abstracts, edited by: Abbasi, A. and Giesen, N., vol. 14 of EGU General Assembly Conference Abstracts, p. 1520, 2012.

Woods, T. N. and Rottman, G. J.: Solar Ultraviolet Variability Over Time Periods of Aeronomic Interest, in: Atmospheres in the Solar System: Comparative Aeronomy, edited by: Mendillo, M., Nagy, A., and Waite, J. H., vol. 130 of AGU Monographs, p. 221, AGU, Washington DC, doi:10.1029/130GM14, 2002.

Woods, T. N., Prinz, D. K., Rottman, G. J., London, J., Crane, P. C., Cebula, R. P., Hilsenrath, E., Brueckner, G. E., Andrews, M. D., White, O. R., VanHoosier, M. E., Floyd, L. E., Herring, L. C., Knapp, B. G., Pankratz, C. K., and Reiser, P. A.: Validation of the UARS solar ultraviolet irradiances: Comparison with the ATLAS 1 and 2 measurements, J. Geophys. Res., 101, 9541-9570, doi:10.1029/96JD00225, 1996.

Woods, T. N., Tobiska, W. K., Rottman, G. J., and Worden, J. R.: Improved solar Lyman $\alpha$ irradiance modeling from 1947 through 1999 based on UARS observations, J. Geophys. Res., 105, 27195-27216, doi:10.1029/2000JA000051, 2000.

Zhong, W., Osprey, S. M., Gray, L. J., and Haigh, J. D.: Influence of the prescribed solar spectrum on calculations of atmospheric temperature, Geophys. Res. Lett., 35, L22813, doi:10.1029/2008GL035993, 2008. 